\title{
Inequalities for generalized Riemann-Liouville fractional integrals of generalized strongly convex functions
}

\author{
Ghulam Farid ${ }^{1}$, Young Chel Kwun ${ }^{2 *}$, Hafsa Yasmeen ${ }^{1}$, Abdullah Akkurt ${ }^{3}$ and Shin Min Kang ${ }^{4,5}$
}

\author{
"Correspondence: \\ yckwun@dau.ac.kr \\ 2Department of Mathematics, \\ Dong-A University, Busan 49315, \\ Korea \\ Full list of author information is \\ available at the end of the article
}

\begin{abstract}
Some new integral inequalities for strongly $(\alpha, h-m)$-convex functions via generalized Riemann-Liouville fractional integrals are established. The outcomes of this paper provide refinements of some fractional integral inequalities for strongly convex, strongly $m$-convex, strongly $(\alpha, m)$-convex, and strongly $(h-m)$-convex functions. Also, the refinements of error estimations of these inequalities are obtained by using two fractional integral identities. Moreover, using a parameter substitution and a constant multiplier, $k$-fractional versions of established inequalities are also given.
\end{abstract}

MSC: 26A51; 26A33; 33E12

Keywords: $(h-m)$-convex function; Strongly $(h-m)$-convex function; $(\alpha, h-m)$-convex function; Strongly $(\alpha, h-m)$-convex function; Hadamard inequality; Riemann-Liouville fractional integrals

\section{Introduction}

Convex function has become an essential notion in the subjects of geometric function theory, mathematical statistics, pure and applied mathematics, physics, mechanics and economics. The generalizations, extensions, and refinements of convex functions are also useful to study classical results for new kinds of functions. The majority of renowned inequalities and properties from various disciplines of mathematics are available in the literature with detailed applications of convexity theory.

Let $I \subseteq \mathbb{R}$ be an interval in $\mathbb{R}$. Then a real-valued function $f: I \rightarrow \mathbb{R}$ is said to be convex function if the following inequality holds:

$$
f(a t+(1-t) b) \leq t f(a)+(1-t) f(b),
$$

for all $a, b \in I$ and $t \in[0,1]$.

The well-known Hadamard inequality describes the convex function in an equivalent way.

(c) The Author(s) 2021. This article is licensed under a Creative Commons Attribution 4.0 International License, which permits use, sharing, adaptation, distribution and reproduction in any medium or format, as long as you give appropriate credit to the original author(s) and the source, provide a link to the Creative Commons licence, and indicate if changes were made. The images or other third party material in this article are included in the article's Creative Commons licence, unless indicated otherwise in a credit line to the material. If material is not included in the article's Creative Commons licence and your intended use is not permitted by statutory regulation or exceeds the permitted use, you will need to obtain permission directly from the copyright holder. To view a copy of this licence, visit http://creativecommons.org/licenses/by/4.0/. 
Let $f: I \rightarrow \mathbb{R}$ be a convex function and $a, b \in I$ where $a<b$. Then the following inequality holds:

$$
f\left(\frac{a+b}{2}\right) \leq \frac{1}{b-a} \int_{a}^{b} f(\xi) d \xi \leq \frac{f(a)+f(b)}{2} .
$$

If the function $f$ is concave on $I$, then the inequality in (1.2) holds in reverse order. This inequality provides lower and upper estimates of the integral mean value of a convex function. The interest of many mathematicians was evoked by this inequality, and several generalizations, extensions, and variants of this inequality have been obtained. In the last two decades it has been remarkably studied, and a lot of papers have been published, see $[1,11,13,14,17,24,29-34]$ and the references therein. Fractional integral inequalities play an important role in mathematics as well as in other areas of mathematics because of their wide applications to establishing the uniqueness of solutions. These solutions can be obtained for certain fractional partial differential equations.

The main objective of this research is to obtain a few versions of the Hadamard inequality for generalized Riemann-Liouville fractional integrals. To achieve this goal, we employ the definition of strongly $(\alpha, h-m)$ convex functions. The refinements of their error estimations are also established. Taking into account parameter substitution and a constant multiplier, $k$-fractional versions of Hadamard inequalities and their estimations for strongly $(\alpha, h-m)$-convex function are proved. In the course of this study, results obtained are a unification and generalization of the comparable results in the literature on Hadamard inequalities. Next, we give the definition of strongly $(\alpha, h-m)$-convex function as follows.

Definition 1 ([35]) Let $J \subseteq \mathbb{R}$ be an interval containing $(0,1)$, and let $h: J \rightarrow \mathbb{R}$ be a nonnegative function. A function $f:[0, b] \rightarrow \mathbb{R}$ is called strongly $(\alpha, h-m)$-convex function with modulus $\lambda \geq 0$, if $f$ is nonnegative and for all $x, y \in[0, b], t \in(0,1), m \in(0,1]$, we have the inequality

$$
f(x t+m(1-t) y) \leq h\left(t^{\alpha}\right) f(x)+m h\left(1-t^{\alpha}\right) f(y)-m \lambda h\left(t^{\alpha}\right) h\left(1-t^{\alpha}\right)|y-x|^{2} .
$$

Fractional calculus is the study of derivatives and integrals of fractional order. Its history is nearly as old as the history of classical calculus. Nevertheless, it has gained the popularity and importance in extensive fields of science and engineering. This field has been widely adopted by many scholars. Recently, motivated by the classical RiemannLiouville fractional integral operators, researchers have defined different integral operators, see $[4,12,25]$. The Riemann-Liouville fractional integral operator is defined as follows.

Definition 2 Let $f \in L_{1}[a, b]$. Then left-sided and right-sided Riemann-Liouville fractional integrals of a function $f$ of order $\mu$, where $\Re(\mu)>0$, are defined by

$$
I_{a^{+}}^{\mu} f(x)=\frac{1}{\Gamma(\mu)} \int_{a}^{x}(x-t)^{\mu-1} f(t) d t, \quad x>a,
$$

and

$$
I_{b-}^{\mu} f(x)=\frac{1}{\Gamma(\mu)} \int_{x}^{b}(t-x)^{\mu-1} f(t) d t, \quad x<b .
$$


Sarikaya et al. [27, 28] elegantly obtained the following fractional integral inequalities of Hadamard type by using the Riemann-Liouville fractional integrals.

Theorem 1 ([27]) Let $f:[a, b] \rightarrow \mathbb{R}$ be a positive function with $0 \leq a<b$ and $f \in L_{1}[a, b]$. Iff is a convex function on $[a, b]$, then the following fractional integral inequality holds:

$$
f\left(\frac{a+b}{2}\right) \leq \frac{\Gamma(\mu+1)}{2(b-a)^{\mu}}\left[I_{a^{+}}^{\mu} f(b)+I_{b^{-}}^{\mu} f(a)\right] \leq \frac{f(a)+f(b)}{2}
$$

with $\mu>0$.

Theorem 2 ([28]) Under the assumptions of Theorem 1, the following fractional integral inequality holds:

$$
f\left(\frac{a+b}{2}\right) \leq \frac{2^{\mu-1} \Gamma(\mu+1)}{(b-a)^{\mu}}\left[I_{\left(\frac{a+b}{2}\right)+}^{\mu} f(b)+I_{\left(\frac{a+b}{2}\right)}^{\mu} f(a)\right] \leq \frac{f(a)+f(b)}{2}
$$

with $\mu>0$.

Theorem 3 ([27]) Let $f:[a, b] \rightarrow \mathbb{R}$ be a differentiable mapping on $(a, b)$ with $a<b$. If $\left|f^{\prime}\right|$ is convex on $[a, b]$, then the following fractional integral inequality holds:

$$
\left|\frac{f(a)+f(b)}{2}-\frac{\Gamma(\mu+1)}{2(b-a)^{\mu}}\left[I_{a^{+}}^{\mu} f(b)+I_{b^{-}}^{\mu} f(a)\right]\right| \leq \frac{b-a}{2(\mu+1)}\left(1-\frac{1}{2^{\mu}}\right)\left[\left|f^{\prime}(a)\right|+\left|f^{\prime}(b)\right|\right] .
$$

The definition of $k$-fractional integral is stated as follows.

Definition 3 ([23]) Let $f \in L_{1}[a, b]$. Then $k$-fractional Riemann-Liouville integrals of order $\mu$, where $\Re(\mu)>0, k>0$, are defined by

$$
{ }_{k} I_{a^{+}}^{\mu} f(x)=\frac{1}{k \Gamma_{k}(\mu)} \int_{a}^{x}(x-t)^{\frac{\mu}{k}-1} f(t) d t, \quad x>a,
$$

and

$$
{ }_{k} I_{b}^{\mu} f(x)=\frac{1}{k \Gamma_{k}(\mu)} \int_{x}^{b}(t-x)^{\frac{\mu}{k}-1} f(t) d t, \quad x<b,
$$

where $\Gamma_{k}(\cdot)$ is defined as follows:

$$
\Gamma_{k}(\mu)=\int_{0}^{\infty} t^{\mu-1} e^{-\frac{t^{k}}{k}} d t, \quad \Re(\mu)>0 .
$$

The definition of generalized fractional integrals by a monotonically increasing function is given as follows.

Definition 4 ([15]) Let $f:[a, b] \rightarrow \mathbb{R}$ be an integrable function. Also let $\psi$ be an increasing and positive monotone function on $(a, b]$ having a continuous derivative $\psi^{\prime}$ on $(a, b)$. The left-sided and right-sided fractional integrals of a function $f$ with respect to another function $\psi$ on $[a, b]$ of order $\mu$, where $\Re(\mu)>0$, are defined by

$$
I_{a^{+}}^{\mu, \psi} f(x)=\frac{1}{\Gamma(\mu)} \int_{a}^{x} \psi^{\prime}(t)(\psi(x)-\psi(t))^{\mu-1} f(t) d t, \quad x>a,
$$




$$
I_{b^{-}}^{\mu, \psi} f(x)=\frac{1}{\Gamma(\mu)} \int_{x}^{b} \psi^{\prime}(t)(\psi(t)-\psi(x))^{\mu-1} f(t) d t, \quad x<b .
$$

The $k$-analogue of generalized fractional integrals is defined as follows.

Definition 5 ([2]) Let $f:[a, b] \rightarrow \mathbb{R}$ be an integrable function. Also, let $\psi$ be an increasing and positive monotone function on $(a, b]$ having a continuous derivative $\psi^{\prime}$ on $(a, b)$. The left-sided and right-sided fractional integrals of a function $f$ with respect to another function $\psi$ on $[a, b]$ of order $\mu$, where $\Re(\mu)>0, k>0$, are defined by

$$
\begin{aligned}
& { }_{k} I_{a^{+}}^{\mu, \psi} f(x)=\frac{1}{k \Gamma_{k}(\mu)} \int_{a}^{x} \psi^{\prime}(t)(\psi(x)-\psi(t))^{\frac{\mu}{k}-1} f(t) d t, \quad x>a, \\
& { }_{k} I_{b^{-}}^{\mu, \psi} f(x)=\frac{1}{k \Gamma_{k}(\mu)} \int_{x}^{b} \psi^{\prime}(t)(\psi(t)-\psi(x))^{\frac{\mu}{k}-1} f(t) d t, \quad x<b .
\end{aligned}
$$

Using the fact $\Gamma_{k}(\mu)=k^{\frac{\mu}{k}-1} \Gamma\left(\frac{\mu}{k}\right)$ in (1.10) and (1.11) after replacing $\mu$ with $\frac{\mu}{k}$, we get

$$
\begin{aligned}
& k^{\frac{-\mu}{k}} I_{a^{+}}^{\frac{\mu}{k}, \psi} f(x)={ }_{k} I_{a^{+}}^{\mu, \psi} f(x), \\
& k^{\frac{-\mu}{k}} I_{b^{-}}^{\mu, \psi} f(x)={ }_{k} I_{b^{-}}^{\mu, \psi} f(x) .
\end{aligned}
$$

For more details on the above defined fractional integrals, we refer the readers to see $[21,26]$. In the upcoming section, Hadamard inequalities for strongly $(\alpha, h-m)$-convex function via (1.10) and (1.11) are derived. Also, we give refinements of many fractional versions of Hadamard inequalities proved in [3, 5-10, 16, 18, 19, 27, 28]. In Sect. 3, by using two different fractional integral identities, error bounds of the established inequalities are given. Section 4 contains $k$-fractional versions of Hadamard inequalities and their estimations for strongly $(\alpha, h-m)$-convex function.

\section{Fractional versions of Hadamard inequalities for strongly $(\alpha, h-m)$-convex \\ function}

Theorem 4 Let $f:[a, b] \rightarrow \mathbb{R}$ be a positive function with $0 \leq a<m b$ and $f \in L_{1}[a, b]$. Also, suppose that $f$ is a strongly $(\alpha, h-m)$-convex function on $[a, b]$ with modulus $c \geq 0, \psi$ is a positive strictly increasing function having continuous derivative $\psi^{\prime}$ on $(a, b)$. If $[a, b] \subset$ $\operatorname{Range}(\psi)$ and $(\alpha, m) \in(0,1]^{2}$, then the following fractional integral inequality holds:

$$
\begin{aligned}
f\left(\frac{a+m b}{2}\right)+\frac{c m h\left(\frac{1}{2^{\alpha}}\right) h\left(\frac{2^{\alpha}-1}{2^{\alpha}}\right)}{(\mu+1)(\mu+2)}\left[\mu(\mu+1)(b-a)^{2}+2\left(\frac{a}{m}-m b\right)^{2}\right. \\
\left.\quad+2 \mu(b-a)\left(\frac{a}{m}-m b\right)\right] \\
\leq \frac{\Gamma(\mu+1)}{(m b-a)^{\mu}}\left[h\left(\frac{1}{2^{\alpha}}\right) I_{\psi^{-1}(a)^{+}}^{\mu, \psi}(f \circ \psi)\left(\psi^{-1}(m b)\right)\right. \\
\left.\quad+m^{\mu+1} h\left(\frac{2^{\alpha}-1}{2^{\alpha}}\right) I_{\psi^{-1}(b)^{-}}^{\mu, \psi}(f \circ \psi)\left(\psi^{-1}\left(\frac{a}{m}\right)\right)\right] \\
\leq \mu\left[h\left(\frac{1}{2^{\alpha}}\right) f(a)+m h\left(\frac{2^{\alpha}-1}{2^{\alpha}}\right) f(b)\right] \int_{0}^{1} h\left(t^{\alpha}\right) t^{\mu-1} d t \\
\quad+m \mu\left[h\left(\frac{1}{2^{\alpha}}\right) f(b)+m h\left(\frac{2^{\alpha}-1}{2^{\alpha}}\right) f\left(\frac{a}{m^{2}}\right)\right] \int_{0}^{1} t^{\mu-1} h\left(1-t^{\alpha}\right) d t
\end{aligned}
$$




$$
-c m \mu\left[h\left(\frac{1}{2^{\alpha}}\right)(b-a)^{2}+m h\left(\frac{2^{\alpha}-1}{2^{\alpha}}\right)\left(b-\frac{a}{m^{2}}\right)^{2}\right] \int_{0}^{1} t^{\mu-1} h\left(t^{\alpha}\right) h\left(1-t^{\alpha}\right) d t
$$

with $\mu>0$.

Proof Since the function $f:[a, b] \rightarrow \mathbb{R}$ is a strongly $(\alpha, h-m)$-convex function, for $x, y \in$ $[a, b]$, we have

$$
f\left(\frac{x+m y}{2}\right) \leq h\left(\frac{1}{2^{\alpha}}\right) f(x)+m h\left(\frac{2^{\alpha}-1}{2^{\alpha}}\right) f(y)-\operatorname{cmh}\left(\frac{1}{2^{\alpha}}\right) h\left(\frac{2^{\alpha}-1}{2^{\alpha}}\right)|y-x|^{2} .
$$

By setting $x=a t+m(1-t) b, y=\frac{a}{m}(1-t)+b t$ and integrating the resulting inequality over the interval $[0,1]$ after multiplying by $t^{\mu-1}$, we get

$$
\begin{aligned}
\frac{1}{\mu} f\left(\frac{a+m b}{2}\right) \leq & h\left(\frac{1}{2^{\alpha}}\right) \int_{0}^{1} f(a t+m(1-t) b) t^{\mu-1} d t \\
& +m h\left(\frac{2^{\alpha}-1}{2^{\alpha}}\right) \int_{0}^{1} f\left(\frac{a}{m}(1-t)+b t\right) t^{\mu-1} d t-\frac{c m h\left(\frac{1}{2^{\alpha}}\right) h\left(\frac{2^{\alpha}-1}{2^{\alpha}}\right)}{\mu(\mu+1)(\mu+2)} \\
& \times\left[\mu(\mu+1)(b-a)^{2}+2\left(\frac{a}{m}-m b\right)^{2}+2 \mu(b-a)\left(\frac{a}{m}-m b\right)\right]
\end{aligned}
$$

Now, let $u \in[a, b]$ such that $\psi(u)=a t+m(1-t) b$, that is, $t=\frac{m b-\psi(u)}{m b-a}$, and let $v \in[a, b]$ such that $\psi(v)=\frac{a}{m}(1-t)+b t$, that is, $t=\frac{\psi(v)-\frac{a}{m}}{b-\frac{a}{m}}$ in (2.3). Then, by applying Definition 4 and multiplying by $\mu$, we get the following inequality:

$$
\begin{aligned}
f\left(\frac{a+m b}{2}\right) \leq & \frac{\Gamma(\mu+1)}{(m b-a)^{\mu}}\left[h\left(\frac{1}{2^{\alpha}}\right) I_{\psi^{-1}(a)^{+}}^{\mu, \psi}(f \circ \psi)\left(\psi^{-1}(m b)\right)\right. \\
& \left.+m^{\mu+1} h\left(\frac{2^{\alpha}-1}{2^{\alpha}}\right) I_{\psi^{-1}(b)^{-}}^{\mu, \psi}(f \circ \psi)\left(\psi^{-1}\left(\frac{a}{m}\right)\right)\right] \\
& -\frac{c m h\left(\frac{1}{2^{\alpha}}\right) h\left(\frac{2^{\alpha}-1}{2^{\alpha}}\right)}{(\mu+1)(\mu+2)}\left[\mu(\mu+1)(b-a)^{2}\right. \\
& \left.+2\left(\frac{a}{m}-m b\right)^{2}+2 \mu(b-a)\left(\frac{a}{m}-m b\right)\right] .
\end{aligned}
$$

Hence the first inequality of (2.1) is obtained. On the other hand, $f$ is a strongly $(\alpha, h-m)$ convex function with modulus $c$, we have the following inequality:

$$
\begin{aligned}
& h\left(\frac{1}{2^{\alpha}}\right) f(a t+m(1-t) b)+m h\left(\frac{2^{\alpha}-1}{2^{\alpha}}\right) f\left(\frac{a}{m}(1-t)+b t\right) \\
& \leq \quad h\left(t^{\alpha}\right)\left[h\left(\frac{1}{2^{\alpha}}\right) f(a)+m h\left(\frac{2^{\alpha}-1}{2^{\alpha}}\right) f(b)\right] \\
& \quad+m h\left(1-t^{\alpha}\right)\left[h\left(\frac{1}{2^{\alpha}}\right) f(b)+m h\left(\frac{2^{\alpha}-1}{2^{\alpha}}\right) f\left(\frac{a}{m^{2}}\right)\right] \\
& \quad-\operatorname{cmh}\left(t^{\alpha}\right) h\left(1-t^{\alpha}\right)\left[h\left(\frac{1}{2^{\alpha}}\right)(b-a)^{2}+m h\left(\frac{2^{\alpha}-1}{2^{\alpha}}\right)\left(b-\frac{a}{m^{2}}\right)^{2}\right] .
\end{aligned}
$$


Multiplying inequality (2.4) by $t^{\mu-1}$ and then integrating over the interval $[0,1]$, we get

$$
\begin{aligned}
h\left(\frac{1}{2^{\alpha}}\right) & \int_{0}^{1} f(t a+m(1-t) b) t^{\mu-1} d t \\
& +m h\left(\frac{2^{\alpha}-1}{2^{\alpha}}\right) \int_{0}^{1} f\left(\frac{a}{m}(1-t)+t b\right) t^{\mu-1} d t \\
\leq & {\left[h\left(\frac{1}{2^{\alpha}}\right) f(a)+m h\left(\frac{2^{\alpha}-1}{2^{\alpha}}\right) f(b)\right] \int_{0}^{1} h\left(t^{\alpha}\right) t^{\mu-1} d t } \\
& +\left[h\left(\frac{1}{2^{\alpha}}\right) f(b)+m h\left(\frac{2^{\alpha}-1}{2^{\alpha}}\right) f\left(\frac{a}{m^{2}}\right)\right] \int_{0}^{1} t^{\mu-1} h\left(1-t^{\alpha}\right) d t \\
& -c m\left[h\left(\frac{1}{2^{\alpha}}\right)(b-a)^{2}+m h\left(\frac{2^{\alpha}-1}{2^{\alpha}}\right)\left(b-\frac{a}{m^{2}}\right)^{2}\right] \int_{0}^{1} h\left(t^{\alpha}\right) h\left(1-t^{\alpha}\right) t^{\mu-1} d t .
\end{aligned}
$$

Using substitutions in (2.5) as considered in (2.3) leads to the second inequality of (2.1).

\section{Remark 1}

(i) If $\alpha=m=1$ and $h(t)=t$ in (2.1), we get the inequality stated in [10, Corollary 1 ].

(ii) If $\alpha=1, c=0$ and take $\psi$ as the identity function in (2.1), we get the inequality stated in [6, Corollary 2.2].

(iii) If $\alpha=m=1, c=0, h(t)=t$ and take $\psi$ as the identity function in (2.1), we get Theorem 1.

(iv) If $\alpha=\mu=m=1, h(t)=t, c=0$ and take $\psi$ as the identity function in (2.1), we get Hadamard inequality.

(v) If $\alpha=m=1, c=0$, and $h(t)=t$ in (2.1), we get the inequality stated in [18, Theorem 2.1].

(vi) If $\alpha=\mu=m=1, h(t)=t$ and take $\psi$ as the identity function in (2.1), we get the inequality stated in [19, Theorem 6].

(vii) If $\alpha=1, h(t)=t$ and take $\psi$ as the identity function in (2.1), we get the inequality stated in [5, Theorem 6].

(viii) If $\alpha=1, h(t)=t, c=0$ and take $\psi$ as the identity function in (2.1), we get the inequality stated in [8, Theorem 2.1].

(ix) If $h(t)=t$ in (2.1), we get the inequality stated in [9, Corollary 2].

Corollary 1 If $\alpha=1$ in (2.1), then the following inequality holds for strongly $(h-m)$-convex function:

$$
\begin{aligned}
f\left(\frac{a+m b}{2}\right)+\frac{c m h^{2}\left(\frac{1}{2}\right)}{(\mu+1)(\mu+2)}\left[\mu(\mu+1)(b-a)^{2}+2\left(\frac{a}{m}-m b\right)^{2}\right. \\
\left.\quad+2 \mu(b-a)\left(\frac{a}{m}-m b\right)\right] \\
\leq \frac{h\left(\frac{1}{2}\right) \Gamma(\mu+1)}{(m b-a)^{\mu}}\left[I_{\psi^{-1}(a)^{+}}^{\mu, \psi}(f \circ \psi)\left(\psi^{-1}(m b)\right)+m^{\mu+1} I_{\psi^{-1}(b)^{-}}^{\mu, \psi}(f \circ \psi)\left(\psi^{-1}\left(\frac{a}{m}\right)\right)\right] \\
\leq \mu h\left(\frac{1}{2}\right)[f(a)+m f(b)] \int_{0}^{1} h(t) t^{\mu-1} d t \\
\quad+m \mu h\left(\frac{1}{2}\right)\left[f(b)+m f\left(\frac{a}{m^{2}}\right)\right] \int_{0}^{1} h(1-t) t^{\mu-1} d t
\end{aligned}
$$




$$
-\operatorname{cm} \mu h\left(\frac{1}{2}\right)\left[(b-a)^{2}+m\left(b-\frac{a}{m^{2}}\right)^{2}\right] \int_{0}^{1} t^{\mu-1} h(t) h(1-t) d t
$$

and if $c=0$, then the result for $(h-m)$-convex function can be obtained.

Corollary 2 If $\alpha=1$ and $h(t)=t$ in (2.1), then the following inequality for strongly $m$ convex function holds:

$$
\begin{aligned}
& f\left(\frac{a+m b}{2}\right)+\frac{c m}{4(\mu+1)(\mu+2)}\left[\mu(\mu+1)(b-a)^{2}+2\left(\frac{a}{m}-m b\right)^{2}\right. \\
& \left.\quad+2 \mu(b-a)\left(\frac{a}{m}-m b\right)\right] \\
& \quad \leq \frac{\Gamma(\mu+1)}{2(m b-a)^{\mu}}\left[I_{\psi^{-1}(a)^{+}}^{\mu, \psi}(f \circ \psi)\left(\psi^{-1}(m b)\right)+m^{\mu+1} I_{\psi^{-1}(b)^{-}}^{\mu, \psi}(f \circ \psi)\left(\psi^{-1}\left(\frac{a}{m}\right)\right)\right] \\
& \quad \leq \frac{\mu f(a)}{2(\mu+1)}+\frac{m f(b)}{2}+\frac{m^{2} f\left(\frac{a}{m^{2}}\right)}{(\mu+1)}-\frac{c m \mu\left[(b-a)^{2}+m\left(b-\frac{a}{m^{2}}\right)^{2}\right]}{2(\mu+1)(\mu+2)}
\end{aligned}
$$

and if $c=0$ in the above inequality, then the result for $m$-convex function can be obtained.

Remark 2 For $c>0$, all the results stated in the above corollaries and remark provide the refinements.

Theorem 5 Under the assumptions of Theorem 4, the following fractional integral inequality holds:

$$
\begin{aligned}
f\left(\frac{a+m b}{2}\right)+\frac{c m h\left(\frac{1}{2^{\alpha}}\right) h\left(\frac{2^{\alpha}-1}{2^{\alpha}}\right)}{4(\mu+1)(\mu+2)}\left[\mu(\mu+1)(b-a)^{2}+\left(\frac{a}{m}-m b\right)^{2}\left(\mu^{2}+5 \mu+8\right)\right. \\
\left.\quad+2 \mu(b-a)\left(\frac{a}{m}-m b\right)(\mu+3)\right] \\
\leq \frac{2^{\mu} \Gamma(\mu+1)}{(m b-a)^{\mu}}\left[h\left(\frac{1}{2^{\alpha}}\right) I_{\psi^{-1}\left(\frac{a+m b}{2}\right)^{+}}^{\mu, \psi}(f)\left(\psi^{-1}(m b)\right)\right. \\
\left.\quad+m^{\mu+1} h\left(\frac{2^{\alpha}-1}{2^{\alpha}}\right) I_{\psi^{-1}\left(\frac{a+m b}{2 m}\right)^{-}}^{\mu, \psi}(f)\left(\psi^{-1}\left(\frac{a}{m}\right)\right)\right] \\
\leq \quad \mu\left[h\left(\frac{1}{2^{\alpha}}\right) f(a)+m h\left(\frac{2^{\alpha}-1}{2^{\alpha}}\right) f(b)\right] \int_{0}^{1} h\left(\frac{t^{\alpha}}{2^{\alpha}}\right) t^{\mu-1} d t \\
\quad+\mu m\left[h\left(\frac{1}{2^{\alpha}}\right) f(b)+m h\left(\frac{2^{\alpha}-1}{2^{\alpha}}\right) f\left(\frac{a}{m^{2}}\right)\right] \int_{0}^{1} h\left(\frac{2^{\alpha}-t^{\alpha}}{2^{\alpha}}\right) t^{\mu-1} d t \\
\quad-\mu c m\left[h\left(\frac{1}{2^{\alpha}}\right)(b-a)^{2}+m h\left(\frac{2^{\alpha}-1}{2^{\alpha}}\right)\left(b-\frac{a}{m^{2}}\right)^{2}\right] \\
\quad \times \int_{0}^{1} h\left(\frac{t^{\alpha}}{2^{\alpha}}\right) h\left(\frac{2^{\alpha}-t^{\alpha}}{2^{\alpha}}\right) t^{\mu-1} d t
\end{aligned}
$$

with $\mu>0$. 
Proof Let $x=\frac{a t}{2}+m\left(\frac{2-t}{2}\right) b, y=\frac{a}{m}\left(\frac{2-t}{2}\right)+\frac{b t}{2}$ in (2.2), and integrating the resulting inequality over $[0,1]$ after multiplying by $t^{\mu-1}$, we get

$$
\begin{aligned}
\frac{1}{\mu} f\left(\frac{a+m b}{2}\right) \leq & h\left(\frac{1}{2^{\alpha}}\right) \int_{0}^{1} f\left(\frac{a t}{2}+m\left(\frac{2-t}{2}\right) b\right) t^{\mu-1} d t \\
& +m h\left(\frac{2^{\alpha}-1}{2^{\alpha}}\right) \int_{0}^{1} f\left(\frac{a}{m}\left(\frac{2-t}{2}\right)+\frac{b t}{2}\right) t^{\mu-1} d t \\
& -\frac{c m h\left(\frac{1}{2^{\alpha}}\right) h\left(\frac{2^{\alpha}-1}{2^{\alpha}}\right)}{4 \mu(\mu+1)(\mu+2)}\left[\mu(\mu+1)(b-a)^{2}\right. \\
& \left.+\left(\frac{a}{m}-m b\right)^{2}\left(\mu^{2}+5 \mu+8\right)+2 \mu(b-a)\left(\frac{a}{m}-m b\right)(\mu+3)\right] .
\end{aligned}
$$

Let $u \in[a, b]$ such that $\psi(u)=\frac{a t}{2}+m\left(\frac{2-t}{2}\right) b$, that is, $t=\frac{2(m b-\psi(u))}{m b-a}$ and $v \in[a, b]$ such that $\psi(v)=\frac{a}{m}\left(\frac{2-t}{2}\right)+\frac{b t}{2}$, that is, $t=\frac{2\left(\psi(v)-\frac{a}{m}\right)}{b-\frac{a}{m}}$ in (2.7), then by applying Definition 4 and multiplying by $\mu$, we get the following inequality:

$$
\begin{aligned}
f\left(\frac{a+m b}{2}\right) \leq & \frac{2^{\mu} \Gamma(\mu+1)}{(m b-a)^{\mu}}\left[h\left(\frac{1}{2^{\alpha}}\right) I_{\psi^{-1}\left(\frac{a+m b}{2}\right)^{+}}^{\mu, \psi}(f \circ \psi)\left(\psi^{-1}(m b)\right)\right. \\
& \left.+m^{\mu+1} h\left(\frac{2^{\alpha}-1}{2^{\alpha}}\right) I_{\psi^{-1}\left(\frac{a+m b}{2 m}\right)^{-}}^{\mu, \psi}(f \circ \psi)\left(\psi^{-1}\left(\frac{a}{m}\right)\right)\right] \\
& -\frac{c m h\left(\frac{1}{2^{\alpha}}\right) h\left(\frac{2^{\alpha}-1}{2^{\alpha}}\right)}{4(\mu+1)(\mu+2)}\left[\mu(\mu+1)(b-a)^{2}\right. \\
& \left.+\left(\frac{a}{m}-m b\right)^{2}\left(\mu^{2}+5 \mu+8\right)+2 \mu(b-a)\left(\frac{a}{m}-m b\right)(\mu+3)\right] .
\end{aligned}
$$

Hence the first inequality of (2.6) is obtained. Since $f$ is a strongly $(\alpha, h-m)$-convex function on $[a, b]$ with modulus $c$, we have the following inequality:

$$
\begin{aligned}
& h\left(\frac{1}{2^{\alpha}}\right) f\left(\frac{a t}{2}+m\left(\frac{2-t}{2}\right) b\right)+m h\left(\frac{2^{\alpha}-1}{2^{\alpha}}\right) f\left(\frac{a}{m}\left(\frac{2-t}{2}\right)+\frac{b t}{2}\right) \\
& \leq h\left(\frac{t^{\alpha}}{2^{\alpha}}\right)\left[h\left(\frac{1}{2^{\alpha}}\right) f(a)+m h\left(\frac{2^{\alpha}-1}{2^{\alpha}}\right) f(b)\right]+m h\left(\frac{2^{\alpha}-t^{\alpha}}{2^{\alpha}}\right)\left[h\left(\frac{1}{2^{\alpha}}\right) f(b)\right. \\
&\left.+m h\left(\frac{2^{\alpha}-1}{2^{\alpha}}\right) f\left(\frac{a}{m^{2}}\right)\right]-\operatorname{cmh}\left(\frac{t^{\alpha}}{2^{\alpha}}\right) h\left(\frac{2^{\alpha}-t^{\alpha}}{2^{\alpha}}\right)\left[(b-a)^{2}+m\left(b-\frac{a}{m^{2}}\right)^{2}\right] .
\end{aligned}
$$

Multiplying (2.8) by $t^{\mu-1}$ and then integrating over $[0,1]$, we get

$$
\begin{aligned}
& h\left(\frac{1}{2^{\alpha}}\right) \int_{0}^{1} f\left(\frac{a t}{2}+m\left(\frac{2-t}{2}\right) b\right) t^{\mu-1} d t \\
& \quad+m h\left(\frac{2^{\alpha}-1}{2^{\alpha}}\right) \int_{0}^{1} f\left(\frac{a}{m}\left(\frac{2-t}{2}\right)+\frac{b t}{2}\right) t^{\mu-1} d t \\
& \leq\left[h\left(\frac{1}{2^{\alpha}}\right) f(a)+m h\left(\frac{2^{\alpha}-1}{2^{\alpha}}\right) f(b)\right] \int_{0}^{1} h\left(\frac{t^{\alpha}}{2^{\alpha}}\right) t^{\mu-1} d t+m\left(h\left(\frac{1}{2^{\alpha}}\right) f(b)\right.
\end{aligned}
$$




$$
\begin{aligned}
& \left.+m h\left(\frac{2^{\alpha}-1}{2^{\alpha}}\right) f\left(\frac{a}{m^{2}}\right)\right) \int_{0}^{1} h\left(\frac{2^{\alpha}-t^{\alpha}}{2^{\alpha}}\right) t^{\mu-1} d t-c m\left[(b-a)^{2}+m\left(b-\frac{a}{m^{2}}\right)^{2}\right] \\
& \times \int_{0}^{1} h\left(\frac{t^{\alpha}}{2^{\alpha}}\right) h\left(\frac{2^{\alpha}-t^{\alpha}}{2^{\alpha}}\right) t^{\mu-1} d t .
\end{aligned}
$$

Using the substitutions in (2.9) as considered in (2.7) leads to the second inequality of (2.6).

\section{Remark 3}

(i) If $\alpha=m=1$ and $h(t)=t$ in (2.6), we get the inequality stated in [10, Corollary 4].

(ii) If $\alpha=m=1, h(t)=t, c=0$ and take $\psi$ as the identity function in (2.6), we get Theorem 2 .

(iii) If $\alpha=\mu=m=1, h(t)=t, c=0$ and take $\psi$ as the identity function in (2.6), we get the Hadamard inequality.

(iv) If $h(t)=t$ in (2.6), we get the inequality stated in [9, Corollary 5].

(v) If $\alpha=1, h(t)=t$ and take $\psi$ as the identity function in (2.6), we get the inequality stated in [5, Theorem 7].

(vi) If $\alpha=1, h(t)=t, c=0$ and take $\psi$ as the identity function in (2.6), we get the inequality stated in [7, Theorem 2.1].

(vii) If $\alpha=\mu=m=1, h(t)=t$ and $\psi$ as the identity function in (2.6), we get the inequality stated in [19, Theorem 6].

(viii) If $\alpha=m=1, c=0$, and $h(t)=t$ in (2.6), we get the inequality stated in [22, Lemma 1].

Corollary 3 If $\alpha=1$ in (2.6), then the following inequality holds for strongly $(h-m)$-convex function:

$$
\begin{aligned}
f\left(\frac{a+m b}{2}\right)+\frac{c m h^{2}\left(\frac{1}{2}\right)}{4(\mu+1)(\mu+2)}\left[\mu(\mu+1)(b-a)^{2}+\left(\frac{a}{m}-m b\right)^{2}\left(\mu^{2}+5 \mu+8\right)\right. \\
\left.\quad+2 \mu(b-a)\left(\frac{a}{m}-m b\right)(\mu+3)\right] \\
\leq \quad \frac{2^{\mu} h\left(\frac{1}{2}\right) \Gamma(\mu+1)}{(m b-a)^{\mu}}\left[I_{\psi^{-1}\left(\frac{a+m b}{2}\right)^{+}}^{\mu, \psi}(f)\left(\psi^{-1}(m b)\right)\right. \\
\left.\quad+m^{\mu+1} I_{\psi^{-1}\left(\frac{a+m b}{2 m}\right)^{-}}^{\mu, \psi}(f)\left(\psi^{-1}\left(\frac{a}{m}\right)\right)\right] \\
\leq \quad \mu h\left(\frac{1}{2}\right)[f(a)+m f(b)] \int_{0}^{1} h\left(\frac{t}{2}\right) t^{\mu-1} d t \\
\quad+\mu m h\left(\frac{1}{2}\right)\left[f(b)+m f\left(\frac{a}{m^{2}}\right)\right] \int_{0}^{1} h\left(\frac{2-t}{2}\right) t^{\mu-1} d t \\
\quad-\mu c m h\left(\frac{1}{2}\right)\left[(b-a)^{2}+m\left(b-\frac{a}{m^{2}}\right)^{2}\right] \int_{0}^{1} h\left(\frac{t}{2}\right) h\left(\frac{2-t}{2}\right) t^{\mu-1} d t .
\end{aligned}
$$

If $c=0$ in the above inequality, then the result for $(h-m)$-convex function can be obtained. Also, taking $\psi$ as the identity function in the above inequality, the result for strongly $(h-m)$ convex function via Riemann-Liouville fractional integrals can be obtained. 
Corollary 4 If $\alpha=1$ and $h(t)=t$ in (2.6), then the following inequality holds for strongly m-convex function:

$$
\begin{aligned}
f( & \left.\frac{a+m b}{2}\right)+\frac{c m}{16(\mu+1)(\mu+2)}\left[\mu(\mu+1)(b-a)^{2}+\left(\frac{a}{m}-m b\right)^{2}\left(\mu^{2}+5 \mu+8\right)\right. \\
& \left.+2 \mu(b-a)\left(\frac{a}{m}-m b\right)(\mu+3)\right] \\
\leq & \frac{2^{\mu-1} \Gamma(\mu+1)}{(m b-a)^{\mu}}\left[I_{\psi^{-1}\left(\frac{a+m b}{2}\right)^{+}}^{\mu, \psi} \circ \psi\right)\left(\psi^{-1}(m b)\right) \\
& \left.\quad+m^{\mu+1} I_{\psi^{-1}\left(\frac{a+m b}{2 m}\right)^{-}}^{\mu, \psi}(f \circ \psi)\left(\psi^{-1}\left(\frac{a}{m}\right)\right)\right] \\
\leq & \frac{\mu f(a)}{4(\mu+1)}+\frac{m f(b)}{2}+\frac{m^{2} f\left(\frac{a}{m^{2}}\right)}{4(\mu+1)}-\frac{\mu c m(\mu+3)}{8(\mu+1)(\mu+2)}\left[(b-a)^{2}+m\left(b-\frac{a}{m^{2}}\right)^{2}\right] .
\end{aligned}
$$

If $c=0$ in the above inequality, then the result for m-convex function can be obtained.

Remark 4 For $c>0$, all the results stated in the above corollaries and remark provide the refinements.

\section{Error estimations of Hadamard inequalities for strongly $(\alpha, h-m)$-convex functions}

This section is concerned with the error estimations of Hadamard inequalities for strongly $(\alpha, h-m)$-convex function using integral operators (1.10) and (1.11). The consequences of these inequalities reflect the refinements of error bounds of some fractional integral inequalities for convex, $m$-convex, $(\alpha, m)$-convex, and $(h-m)$-convex functions. We need the following identity to prove our next theorem.

Lemma 1 ([9]) Let $a<b$ and $f:[a, b] \rightarrow \mathbb{R}$ be a differentiable mapping on $(a, b)$. Also, suppose that $f^{\prime} \in L[a, b], \psi$ is a positive strictly increasing function having a continuous derivative $\psi^{\prime}$ on $(a, b)$. If $[a, b] \subset \operatorname{Range}(\psi)$, then the following identity holds for generalized fractional integral operators:

$$
\begin{aligned}
& \frac{f(a)+f(b)}{2}-\frac{\Gamma(\mu+1)}{2(b-a)^{\mu}}\left[I_{\psi^{-1}(a)^{+}}^{\mu, \psi}(f \circ \psi)\left(\psi^{-1}(b)\right)+I_{\psi^{-1}(b)^{-}}^{\mu, \psi}(f \circ \psi)\left(\psi^{-1}(a)\right]\right. \\
& =\frac{b-a}{2} \int_{0}^{1}\left((1-t)^{\mu}-t^{\mu}\right) f^{\prime}(t a+(1-t) b) d t .
\end{aligned}
$$

Theorem 6 Let $f:[a, b] \rightarrow \mathbb{R}$ be a differentiable mapping on $(a, b)$ with $0 \leq a<b$. Also suppose that $\left|f^{\prime}\right|$ is strongly $(\alpha, h-m)$-convex with modulus $c \geq 0, \psi$ is a positive strictly increasing function having continuous derivative $\psi^{\prime}$ on $(a, b)$. If $[a, b] \subset \operatorname{Range}(\psi)$ and $(\alpha, m) \in(0,1]^{2}$, then the following fractional integral inequality holds:

$$
\begin{aligned}
& \left|\frac{f(a)+f(b)}{2}-\frac{\Gamma(\mu+1)}{2(b-a)^{\mu}}\left[I_{\psi^{-1}(a)^{+}}^{\mu, \psi}(f \circ \psi)\left(\psi^{-1}(b)\right)+I_{\psi^{-1}(b)}^{\mu, \psi}(f \circ \psi)\left(\psi^{-1}(a)\right)\right]\right| \\
& \leq \frac{b-a}{2}\left[\left|f^{\prime}(a)\right|\left(\int_{0}^{\frac{1}{2}} h\left(t^{\alpha}\right)\left((1-t)^{\mu}-t^{\mu}\right) d t+\int_{\frac{1}{2}}^{1} h\left(t^{\alpha}\right)\left(t^{\mu}-(1-t)^{\mu}\right) d t\right)\right.
\end{aligned}
$$




$$
\begin{aligned}
& +m\left|f^{\prime}\left(\frac{b}{m}\right)\right|\left(\int_{0}^{\frac{1}{2}} h\left(1-t^{\alpha}\right)\left((1-t)^{\mu}-t^{\mu}\right) d t+\int_{\frac{1}{2}}^{1} h\left(1-t^{\alpha}\right)\left(t^{\mu}-(1-t)^{\mu}\right) d t\right) \\
& -c m\left(\frac{b}{m}-a\right)^{2}\left(\int_{0}^{\frac{1}{2}} h\left(t^{\alpha}\right) h\left(1-t^{\alpha}\right)\left((1-t)^{\mu}-t^{\mu}\right)\right) d t \\
& \left.\left.+\int_{\frac{1}{2}}^{1} h(t) h(1-t)\left(t^{\mu}-(1-t)^{\mu}\right) d t\right)\right]
\end{aligned}
$$

with $\mu>0$.

Proof From Lemma 1, it follows that

$$
\begin{aligned}
& \left|\frac{f(a)+f(b)}{2}-\frac{\Gamma(\mu+1)}{2(b-a)^{\mu}}\left[I_{\psi^{-1}(a)^{+}}^{\mu, \psi}(f \circ \psi)\left(\psi^{-1}(b)\right)+I_{\psi^{-1}(b)^{-}}^{\mu, \psi}(f \circ \psi)\left(\psi^{-1}(b)\right)\right]\right| \\
& \quad \leq \frac{b-a}{2} \int_{0}^{1}\left|(1-t)^{\mu}-t^{\mu}\right| f^{\prime}(a t+(1-t) b) \mid d t .
\end{aligned}
$$

By using the strong $(\alpha, h-m)$-convexity of $\left|f^{\prime}\right|$, we have

$$
\begin{aligned}
\left|f^{\prime}(t a+(1-t) b)\right| \leq & h\left(t^{\alpha}\right)\left|f^{\prime}(a)\right|+m h\left(1-t^{\alpha}\right)\left|f^{\prime}\left(\frac{b}{m}\right)\right| \\
& -\operatorname{cmh}\left(t^{\alpha}\right) h\left(1-t^{\alpha}\right)\left(\frac{b}{m}-a\right)^{2} .
\end{aligned}
$$

Now, using (3.4) in (3.3), we have

$$
\begin{aligned}
& \left|\frac{f(a)+f(b)}{2}-\frac{\Gamma(\mu+1)}{2(b-a)^{\mu}}\left[I_{\psi^{-1}(a)^{+}}^{\mu, \psi}(f \circ \psi)\left(\psi^{-1}(b)\right)+I_{\psi^{-1}(b)}^{\mu, \psi}(f \circ \psi)\left(\psi^{-1}(b)\right)\right]\right| \\
& \leq \frac{b-a}{2} \int_{0}^{1}\left|(1-t)^{\mu}-t^{\mu}\right|\left[h\left(t^{\alpha}\right)\left|f^{\prime}(a)\right|+m h\left(1-t^{\alpha}\right)\left|f^{\prime}\left(\frac{b}{m}\right)\right|\right. \\
& \left.\quad-\operatorname{cmh}\left(t^{\alpha}\right) h\left(1-t^{\alpha}\right)\left(\frac{b}{m}-a\right)^{2}\right] d t \\
& =\frac{b-a}{2}\left[\left|f^{\prime}(a)\right|\left(\int_{0}^{\frac{1}{2}} h\left(t^{\alpha}\right)\left((1-t)^{\mu}-t^{\mu}\right) d t+\int_{\frac{1}{2}}^{1} h\left(t^{\alpha}\right)\left(t^{\mu}-\left(1-t^{\alpha}\right)^{\mu}\right) d t\right)\right. \\
& \quad+m\left|f^{\prime}\left(\frac{b}{m}\right)\right|\left(\int_{0}^{\frac{1}{2}} h\left(1-t^{\alpha}\right)\left((1-t)^{\mu}-t^{\mu}\right) d t+\int_{\frac{1}{2}}^{1} h\left(1-t^{\alpha}\right)\left(t^{\mu}-(1-t)^{\mu}\right) d t\right) \\
& \quad-c m\left(\frac{b}{m}-a\right)^{2}\left(\int_{0}^{\frac{1}{2}} h\left(t^{\alpha}\right) h\left(1-t^{\alpha}\right)\left((1-t)^{\mu}-t^{\mu}\right) d t\right. \\
& \left.\left.\quad+\int_{\frac{1}{2}}^{1} h\left(t^{\alpha}\right) h\left(1-t^{\alpha}\right)\left(t^{\mu}-(1-t)^{\mu}\right) d t\right)\right] .
\end{aligned}
$$

\section{Remark 5}

(i) If $\alpha=m=1$ and $h(t)=t$ in (3.2), we get the inequality stated in [10, Corollary 7].

(ii) If $\alpha=m=1, h(t)=t, c=0$ and take $\psi$ as the identity function in (3.2), we get Theorem 3. 
(iii) If $\alpha=1, h(t)=t$ and take $\psi$ as the identity function in (3.2), we get the inequality stated in [5, Theorem 8].

(iv) If $\alpha=\mu=m=1, h(t)=t, c=0$ and take $\psi$ as the identity function in (3.2), we get the inequality stated in [3, Theorem 2.2].

Corollary 5 If $\alpha=1$ in (3.2), then the following inequality holds for strongly $(h-m)$-convex function:

$$
\begin{aligned}
& \left|\frac{f(a)+f(b)}{2}-\frac{\Gamma(\mu+1)}{2(b-a)^{\mu}}\left[I_{\psi^{-1}(a)^{+}}^{\mu, \psi}(f \circ \psi)\left(\psi^{-1}(b)\right)+I_{\psi^{-1}(b)^{-}}^{\mu, \psi}(f \circ \psi)\left(\psi^{-1}(a)\right)\right]\right| \\
& \leq \frac{b-a}{2}\left[\left|f^{\prime}(a)\right|\left(\int_{0}^{\frac{1}{2}} h(t)\left((1-t)^{\mu}-t^{\mu}\right) d t+\int_{\frac{1}{2}}^{1} h(t)\left(t^{\mu}-(1-t)^{\mu}\right) d t\right)\right. \\
& \quad+m\left|f^{\prime}\left(\frac{b}{m}\right)\right|\left(\int_{0}^{\frac{1}{2}} h(1-t)\left((1-t)^{\mu}-t^{\mu}\right) d t+\int_{\frac{1}{2}}^{1} h(1-t)\left(t^{\mu}-(1-t)^{\mu}\right) d t\right) \\
& \quad-c m\left(\frac{b}{m}-a\right)^{2}\left(\int_{0}^{\frac{1}{2}} h(t) h(1-t)\left((1-t)^{\mu}-t^{\mu}\right)\right) d t \\
& \left.\left.\quad+\int_{\frac{1}{2}}^{1} h(t) h(1-t)\left(t^{\mu}-(1-t)^{\mu}\right) d t\right)\right] .
\end{aligned}
$$

For $c=0$ in the above inequality, the result for $(h-m)$-convex function can be obtained. Moreover, if $\psi$ is an identity function, then the result for $(h-m)$-convex function for Riemann-Liouville fractional integrals can be obtained.

Corollary 6 If $\alpha=1$ and $h(t)=t$ in (3.2), then the following inequality holds for strongly m-convex function:

$$
\begin{aligned}
& \left|\frac{f(a)+f(b)}{2}-\frac{\Gamma(\mu+1)}{2(b-a)^{\mu}}\left[I_{\psi^{-1}(a)^{+}}^{\mu, \psi}(f \circ \psi)\left(\psi^{-1}(b)\right)+I_{\psi^{-1}(b)^{-}}^{\mu, \psi}(f \circ \psi)\left(\psi^{-1}(a)\right)\right]\right| \\
& \quad \leq \frac{b-a}{2(\mu+1)}\left(1-\frac{1}{2^{\mu}}\right)\left(\left|f^{\prime}(a)+m\right| f^{\prime}\left(\frac{b}{m}\right) \mid\right)-\frac{c m\left(\frac{b}{m}-a\right)^{2}(b-a)}{(\mu+2)(\mu+3)}\left(1-\frac{\mu+4}{2^{\mu+2}}\right) .
\end{aligned}
$$

If $c=0$ in the above inequality, then the result for $m$-convex function can be obtained.

Corollary 7 If $\alpha=\mu=m=1, h(t)=t$ and take $\psi$ as the identity function in (3.2), we get the following inequality for strongly convex function:

$$
\left|\frac{f(a)+f(b)}{2}-\frac{1}{(b-a)} \int_{a}^{b} f(v) d v\right| \leq \frac{b-a}{8}\left[\left|f^{\prime}(a)\right|+\left|f^{\prime}(b)\right|\right]-\frac{c(b-a)^{2}}{32}
$$

To prove next two theorems, we need the following identity.

Lemma 2 ([20]) Let $f:[a, b] \rightarrow \mathbb{R}$ be a differentiable mapping on $(a, b)$ such that $f^{\prime} \in$ $L[a, b], \psi$ is a positive increasing function having continuous derivative $\psi^{\prime}$ on $(a, b)$. If $[a, b] \subset \operatorname{Range}(\psi)$ and $m \in(0,1]$, then the following fractional integral identity holds:

$$
\frac{2^{\mu-1} \Gamma(\mu+1)}{(m b-a)^{\mu}}\left[I_{\psi^{-1}\left(\frac{a+m b}{2}\right)^{+}}^{\mu, \psi}(f \circ \psi)\left(\psi^{-1}(m b)\right)\right.
$$




$$
\begin{aligned}
& \left.+m^{\mu+1} I_{\psi^{-1}\left(\frac{a+m b}{2 m}\right)^{-}}^{\mu,}(f \circ \psi)\left(\psi^{-1}\left(\frac{a}{m}\right)\right)\right]-\frac{1}{2}\left[f\left(\frac{a+m b}{2}\right)+m f\left(\frac{a+m b}{2 m}\right)\right] \\
= & \frac{m b-a}{4}\left[\int_{0}^{1} t^{\mu} f^{\prime}\left(\frac{a t}{2}+m\left(\frac{2-t}{2}\right) b\right) d t-\int_{0}^{1} t^{\mu} f^{\prime}\left(\frac{a}{m}\left(\frac{2-t}{2}\right)+\frac{b t}{2}\right) d t\right] .
\end{aligned}
$$

Theorem 7 Let $f:[a, b] \rightarrow \mathbb{R}$ be a differentiable mapping on $(a, b)$ such that $f^{\prime} \in €[a, b]$. Also, suppose that $\left|f^{\prime}\right|^{q}$ is a strongly $(\alpha, h-m)$-convex function on $[a, b]$ for $q \geq 1, \psi$ is an increasing and positive monotone function on $(a, b]$ having a continuous derivative $\psi^{\prime}$ on $(a, b)$. If $[a, b] \subset \operatorname{Range}(\psi)$ and $(\alpha, m) \in(0,1]^{2}$, then the following fractional integral inequality holds:

$$
\begin{aligned}
& \mid \frac{2^{\mu-1} \Gamma(\mu+1)}{(m b-a)^{\mu}}\left[I_{\psi^{-1}\left(\frac{a+m b}{2}\right)^{+}}^{\mu, \psi}(f \circ \psi)\left(\psi^{-1}(m b)\right)\right. \\
& \left.\quad+m^{\mu+1} I_{\psi^{-1}\left(\frac{a+m b}{2 m}\right)^{-}}^{\mu,}(f \circ \psi)\left(\psi^{-1}\left(\frac{a}{m}\right)\right)\right] \\
& \quad-\frac{1}{2}\left[f\left(\frac{a+m b}{2}\right)+m f\left(\frac{a+m b}{2 m}\right)\right] \mid \\
& \leq \frac{m b-a}{4(\mu+1)^{1-\frac{1}{q}}}\left[\left(\left|f^{\prime}(a)\right|^{q} \int_{0}^{1} h\left(\frac{t^{\alpha}}{2^{\alpha}}\right) t^{\mu} d t\right.\right. \\
& \left.\quad+m\left|f^{\prime}(b)\right|^{q} \int_{0}^{1} h\left(\frac{2^{\alpha}-t^{\alpha}}{2^{\alpha}}\right) t^{\mu} d t-c m(b-a)^{2} \int_{0}^{1} h\left(\frac{t^{\alpha}}{2^{\alpha}}\right) h\left(\frac{2^{\alpha}-t^{\alpha}}{2^{\alpha}}\right) t^{\mu} d t\right)^{\frac{1}{q}} \\
& \quad+\left(m\left|f^{\prime}\left(\frac{a}{m^{2}}\right)\right|^{q} \int_{0}^{1} h\left(\frac{2^{\alpha}-t^{\alpha}}{2^{\alpha}}\right) t^{\mu} d t+\left|f^{\prime}(b)\right|^{q} \int_{0}^{1} h\left(\frac{t^{\alpha}}{2^{\alpha}}\right) t^{\mu} d t\right. \\
& \left.\left.\quad-c m\left(b-\frac{a}{m^{2}}\right)^{2} \int_{0}^{1} h\left(\frac{t^{\alpha}}{2^{\alpha}}\right) h\left(\frac{2^{\alpha}-t^{\alpha}}{2^{\alpha}}\right) t^{\mu} d t\right)^{\frac{1}{q}}\right]
\end{aligned}
$$

with $\mu>0$.

Proof For $q=1$, applying the Lemma 2 and using the strong $(\alpha, h-m)$-convexity of $\left|f^{\prime}\right|$, we have

$$
\begin{aligned}
& \mid \frac{2^{\mu-1} \Gamma(\mu+1)}{(m b-a)^{\mu}}\left[I_{\psi^{-1}\left(\frac{a+m b}{2}\right)^{+}}^{\mu, \psi}(f \circ \psi)\left(\psi^{-1}(m b)\right)+m^{\mu+1} I_{\psi^{-1}\left(\frac{a+m b}{2 m}\right)^{-}}^{\mu, \psi}(f \circ \psi)\left(\psi^{-1}\left(\frac{a}{m}\right)\right)\right] \\
& \quad-\frac{1}{2}\left[f\left(\frac{a+m b}{2}\right)+m f\left(\frac{a+m b}{2 m}\right)\right] \mid \\
& \leq \frac{m b-a}{4}\left[\int_{0}^{1}\left|t^{\mu} f^{\prime}\left(\frac{a t}{2}+m\left(\frac{2-t}{2}\right) b\right)\right| d t+\int_{0}^{1}\left|t^{\mu} f^{\prime}\left(\frac{a}{m}\left(\frac{2-t}{2}\right)+\frac{b t}{2}\right)\right| d t\right] \\
& \leq \frac{m b-a}{4}\left[\left(\left|f^{\prime}(a)\right|+\left|f^{\prime}(b)\right|\right) \int_{0}^{1} h\left(\frac{t^{\alpha}}{2^{\alpha}}\right) t^{\mu} d t\right. \\
& \quad+m\left(\left|f^{\prime}(b)\right|+\left|f^{\prime}\left(\frac{a}{m^{2}}\right)\right|\right) \int_{0}^{1} h\left(\frac{2^{\alpha}-t^{\alpha}}{2^{\alpha}}\right) t^{\mu} d t-c m\left((b-a)^{2}+\left(b-\frac{a}{m^{2}}\right)^{2}\right) \\
& \left.\quad \times \int_{0}^{1} h\left(\frac{t^{\alpha}}{2^{\alpha}}\right) h\left(\frac{2^{\alpha}-t^{\alpha}}{2^{\alpha}}\right) t^{\mu} d t\right] .
\end{aligned}
$$


Now, for $q>1$, we use Lemma 2 and the power mean inequality

$$
\begin{aligned}
& \mid \frac{2^{\mu-1} \Gamma(\mu+1)}{(m b-a)^{\mu}}\left[I_{\psi^{-1}\left(\frac{a+m b}{2}\right)^{+}}^{\mu, \psi}\left(f \circ \psi\left(\psi^{-1}(m b)\right)+m^{\mu+1} I_{\psi^{-1}\left(\frac{a+m b}{2 m}\right)^{-}}^{\mu, \psi}(f \circ \psi)\left(\psi^{-1}\left(\frac{a}{m}\right)\right)\right]\right. \\
& \quad-\frac{1}{2}\left[f\left(\frac{a+m b}{2}\right)+m f\left(\frac{a+m b}{2 m}\right)\right] \mid \\
& \leq \frac{m b-a}{4}\left(\int_{0}^{1} t^{\mu} d t\right)^{1-\frac{1}{q}}\left[\left(\int_{0}^{1} t^{\mu}\left|f^{\prime}\left(\frac{a t}{2}+m\left(\frac{2-t}{2}\right) b\right)\right|^{q} d t\right)^{\frac{1}{q}}\right. \\
& \left.\quad+\left(\int_{0}^{1} t^{\mu}\left|f^{\prime}\left(\frac{a}{m}\left(\frac{2-t}{2}\right)+\frac{b t}{2}\right)\right|^{q} d t\right)^{\frac{1}{q}}\right] \\
& \leq \quad \frac{m b-a}{4}\left[( \mu + 1 ) ^ { 1 - \frac { 1 } { q } } \left[\left(\left.f^{\prime}(a)\right|^{q} \int_{0}^{1} h\left(\frac{t^{\alpha}}{2^{\alpha}}\right) t^{\mu} d t+m\left|f^{\prime}(b)\right|^{q} \int_{0}^{1} h\left(\frac{2^{\alpha}-t^{\alpha}}{2^{\alpha}}\right) t^{\mu} d t\right.\right.\right. \\
& \left.\quad-c m(b-a)^{2} \int_{0}^{1} h\left(\frac{t^{\alpha}}{2^{\alpha}}\right) h\left(\frac{2^{\alpha}-t^{\alpha}}{2^{\alpha}}\right) t^{\mu} d t\right)^{\frac{1}{q}} \\
& \quad+\left(m\left|f^{\prime}\left(\frac{a}{m^{2}}\right)\right| \int_{0}^{1} h\left(\frac{2^{\alpha}-t^{\alpha}}{2^{\alpha}}\right) t^{\mu} d t+\left|f^{\prime}(b)\right|^{q} \int_{0}^{1} h\left(\frac{t^{\alpha}}{2^{\alpha}}\right) t^{\mu} d t\right. \\
& \left.\left.\quad-c m\left(b-\frac{a}{m^{2}}\right)^{2} \int_{0}^{1} h\left(\frac{t^{\alpha}}{2^{\alpha}}\right) h\left(\frac{2^{\alpha}-t^{\alpha}}{2^{\alpha}}\right) t^{\mu} d t\right)^{\frac{1}{q}}\right] .
\end{aligned}
$$

\section{Remark 6}

(i) If $\alpha=m=1$ and $h(t)=t$ in (3.6), we get the inequality stated in [10, Theorem 12].

(ii) If $\alpha=1, h(t)=t$ and take $\psi$ as the identity function in (3.6), we get the inequality stated in [5, Theorem 9].

(iii) If $\alpha=1, h(t)=t, c=0$ and take $\psi$ as the identity function in (3.6), we get the inequality stated in [7, Theorem 2.4].

(iv) If $\alpha=m=1, h(t)=t, c=0$ and take $\psi$ as the identity function in (3.6), we get the inequality stated in [28, Theorem 5].

(v) If $\alpha=\mu=m=q=1, h(t)=t, c=0$ and take $\psi$ as the identity function in (3.6), we get the inequality stated in [16, Theorem 2.2].

(vi) If $\alpha=\mu=m=q=1, h(t)=t$ and take $\psi$ as the identity function in (3.6), we get the inequality stated in [10, Corollary 13].

Corollary 8 If $\alpha=1$ in (3.6), then the following inequality holds for strongly $(h-m)$-convex function:

$$
\begin{aligned}
& \mid \frac{2^{\mu-1} \Gamma(\mu+1)}{(m b-a)^{\mu}}\left[I_{\psi^{-1}\left(\frac{a+m b}{2}\right)^{+}}^{\mu, \psi}(f \circ \psi)\left(\psi^{-1}(m b)\right)+m^{\mu+1} I_{\psi^{-1}\left(\frac{a+m b}{2 m}\right)^{-}}^{\mu, \psi}(f \circ \psi)\left(\psi^{-1}\left(\frac{a}{m}\right)\right)\right] \\
& \quad-\frac{1}{2}\left[f\left(\frac{a+m b}{2}\right)+m f\left(\frac{a+m b}{2 m}\right)\right] \mid \\
& \leq \frac{m b-a}{4(\mu+1)^{\frac{1}{p}}}\left[\left(\left|f^{\prime}(a)\right|^{q} \int_{0}^{1} h\left(\frac{t}{2}\right) t^{\mu} d t+m\left|f^{\prime}(b)\right|^{q} \int_{0}^{1} h\left(\frac{2-t}{2}\right) t^{\mu} d t\right.\right. \\
& \left.\quad-c m(b-a)^{2} \int_{0}^{1} h\left(\frac{t}{2}\right) h\left(\frac{2-t}{2}\right) t^{\mu} d t\right)^{\frac{1}{q}}
\end{aligned}
$$




$$
\begin{aligned}
& +\left(m\left|f^{\prime}\left(\frac{a}{m^{2}}\right)\right|^{q} \int_{0}^{1} h\left(\frac{2-t}{2}\right) t^{\mu} d t+\left|f^{\prime}(b)\right|^{q} \int_{0}^{1} h\left(\frac{t}{2}\right) t^{\mu} d t\right. \\
& \left.\left.-c m\left(b-\frac{a}{m^{2}}\right)^{2} \int_{0}^{1} h\left(\frac{t}{2}\right) h\left(\frac{2-t}{2}\right) t^{\mu} d t\right)^{\frac{1}{q}}\right] .
\end{aligned}
$$

For $c=0$ in the above inequality, the result for $(h-m)$-convex function can be obtained. Moreover, if $\psi$ is the identity function in the above inequality, then the result for $(h-m)$ convex function for Riemann-Liouville fractional integrals can be obtained.

Corollary 9 If $\alpha=1$ and $h(t)=t$ in (3.6), then the following inequality holds for strongly m-convex function:

$$
\begin{aligned}
& \mid \frac{2^{\mu-1} \Gamma(\mu+1)}{(m b-a)^{\mu}}\left[I_{\psi^{-1}\left(\frac{a+m b}{2}\right)^{+}}^{\mu, \psi}(f \circ \psi)\left(\psi^{-1}(m b)\right)+m^{\mu+1} I_{\psi^{-1}\left(\frac{a+m b}{2 m}\right)^{-}}^{\mu, \psi}(f \circ \psi)\left(\psi^{-1}\left(\frac{a}{m}\right)\right)\right] \\
& \quad-\frac{1}{2}\left[f\left(\frac{a+m b}{2}\right)+m f\left(\frac{a+m b}{2 m}\right)\right] \mid \\
& \leq \frac{m b-a}{4(\mu+1)}\left(\frac{1}{2(\mu+2)}\right)^{\frac{1}{q}}\left[\left(\left|f^{\prime}(a)\right|^{q}(\mu+1)+m\left|f^{\prime}(b)\right|^{q}(\mu+3)\right.\right. \\
& \left.\quad-\frac{c m(b-a)^{2}(\mu+1)(\mu+4)}{2(\mu+3)}\right)^{\frac{1}{q}}+\left(m\left|f^{\prime}\left(\frac{a}{m^{2}}\right)\right|^{q}(\mu+3)\right. \\
& \left.\left.\quad+\left|f^{\prime}(b)\right|^{q}(\mu+1)-\frac{c m\left(b-\frac{a}{m^{2}}\right)^{2}(\mu+1)(\mu+4)}{2(\mu+3)}\right)^{\frac{1}{q}}\right]
\end{aligned}
$$

If $c=0$ in the above inequality, then the result for $m$-convex function can be obtained.

Theorem 8 Let $f:[a, b] \rightarrow \mathbb{R}$ be a differentiable mapping on $(a, b)$ with $a<b$. Also, suppose that $\left|f^{\prime}\right|^{q}$ is a strongly $(\alpha, h-m)$-convex function for $q>1, \psi$ is a positive increasing function having continuous derivative $\psi^{\prime}$ on $(a, b)$. If $[a, b] \subset \operatorname{Range}(\psi)$ and $(\alpha, m) \in(0,1]^{2}$, then the following fractional integral inequality holds:

$$
\begin{aligned}
& \mid \frac{2^{\mu-1} \Gamma(\mu+1)}{(m b-a)^{\mu}}\left[I _ { \psi ^ { - 1 } ( \frac { a + m b } { 2 } ) ^ { + } } ^ { \mu , \psi } ( f \circ \psi ) \left(\psi^{-1}(m b)\right.\right. \\
& \left.\quad+m^{\mu+1} I_{\psi^{-1}\left(\frac{a+m b}{2 m}\right)^{-}}^{\mu, \psi}(f \circ \psi)\left(\psi^{-1}\left(\frac{a}{m}\right)\right)\right]-\frac{1}{2}\left[f\left(\frac{a+m b}{2}\right)+m f\left(\frac{a+m b}{2 m}\right)\right] \mid \\
& \leq \frac{m b-a}{4(\mu p+1)^{\frac{1}{p}}}\left[\left(\left|f^{\prime}(a)\right|^{q} \int_{0}^{1} h\left(\frac{t^{\alpha}}{2^{\alpha}}\right) d t+m\left|f^{\prime}(b)\right|^{q} \int_{0}^{1} h\left(\frac{2^{\alpha}-t^{\alpha}}{2^{\alpha}}\right) d t\right.\right. \\
& \left.\quad-c m(b-a)^{2} \int_{0}^{1} h\left(\frac{t^{\alpha}}{2^{\alpha}}\right) h\left(\frac{2^{\alpha}-t^{\alpha}}{2^{\alpha}}\right) d t\right)^{\frac{1}{q}} \\
& \quad+\left(m\left|f^{\prime}\left(\frac{a}{m^{2}}\right)\right|^{q} \int_{0}^{1} h\left(\frac{2^{\alpha}-t^{\alpha}}{2^{\alpha}}\right) d t+\left|f^{\prime}(b)\right|^{q} \int_{0}^{1} h\left(\frac{t^{\alpha}}{2^{\alpha}}\right) d t\right. \\
& \left.\left.\quad-c m\left(b-\frac{a}{m^{2}}\right)^{2} \int_{0}^{1} h\left(\frac{t^{\alpha}}{2^{\alpha}}\right) h\left(\frac{2^{\alpha}-t^{\alpha}}{2^{\alpha}}\right)\right)^{\frac{1}{q}}\right]
\end{aligned}
$$

with $\mu>0$ and $\frac{1}{p}+\frac{1}{q}=1$. 
Proof By applying Lemma 2 and using the property of modulus, we get

$$
\begin{aligned}
& \mid \frac{2^{\mu-1} \Gamma(\mu+1)}{(m b-a)^{\mu}}\left[I_{\psi^{-1}\left(\frac{a+m b}{2}\right)^{+}}^{\mu, \psi}(f \circ \psi)\left(\psi^{-1}(m b)\right)+m^{\mu+1} I_{\psi^{-1}\left(\frac{a+m b}{2 m}\right)^{-}}^{\mu, \psi}(f \circ \psi)\left(\psi^{-1}\left(\frac{a}{m}\right)\right)\right] \\
& \quad-\frac{1}{2}\left[f\left(\frac{a+m b}{2}\right)+m f\left(\frac{a+m b}{2 m}\right)\right] \mid \\
& \quad \leq \frac{m b-a}{4}\left[\int_{0}^{1}\left|t^{\mu} f^{\prime}\left(\frac{a t}{2}+m\left(\frac{2-t}{2}\right) b\right)\right| d t+\int_{0}^{1}\left|t^{\mu} f^{\prime}\left(\frac{a}{m}\left(\frac{2-t}{2}\right)+\frac{b t}{2}\right)\right| d t\right] .
\end{aligned}
$$

Now, applying Hölder's inequality for integrals, we get

$$
\begin{aligned}
& \mid \frac{2^{\mu-1} \Gamma(\mu+1)}{(m b-a)^{\mu}}\left[I_{\psi^{-1}\left(\frac{a+m b}{2}\right)^{+}}^{\mu, \psi}(f \circ \psi)\left(\psi^{-1}(m b)\right)+m^{\mu+1} I_{\psi^{-1}\left(\frac{a+m b}{2 m}\right)^{-}}^{\mu, \psi}(f \circ \psi)\left(\psi^{-1}\left(\frac{a}{m}\right)\right)\right] \\
& \quad-\frac{1}{2}\left[f\left(\frac{a+m b}{2}\right)+m f\left(\frac{a+m b}{2 m}\right)\right] \mid \\
& \leq \frac{m b-a}{4(\mu p+1)^{\frac{1}{p}}}\left[\left(\int_{0}^{1}\left|f^{\prime}\left(\frac{a t}{2}+m\left(\frac{2-t}{2}\right) b\right)\right|^{q} d t\right)^{\frac{1}{q}}\right. \\
& \left.\quad+\left(\int_{0}^{1}\left|f^{\prime}\left(\frac{a}{m}\left(\frac{2-t}{2}\right)+\frac{b t}{2}\right)\right|^{q} d t\right)^{\frac{1}{q}}\right] .
\end{aligned}
$$

Using the strong $(\alpha, h-m)$-convexity of $\left|f^{\prime}\right|^{q}$, we get

$$
\begin{aligned}
& \mid \frac{2^{\mu-1} \Gamma(\mu+1)}{(m b-a)^{\mu}}\left[I_{\psi^{-1}\left(\frac{a+m b}{2}\right)^{+}}^{\mu, \psi}(f \circ \psi)\left(\psi^{-1}(m b)\right)+m^{\mu+1} I_{\psi^{-1}\left(\frac{a+m b}{2 m}\right)^{-}}^{\mu, \psi}(f \circ \psi)\left(\psi^{-1}\left(\frac{a}{m}\right)\right)\right] \\
& \quad-\frac{1}{2}\left[f\left(\frac{a+m b}{2}\right)+m f\left(\frac{a+m b}{2 m}\right)\right] \mid \\
& \leq \frac{m b-a}{4(\mu p+1)^{\frac{1}{p}}}\left[\left(\left|f^{\prime}(a)\right|^{q} \int_{0}^{1} h\left(\frac{t^{\alpha}}{2^{\alpha}}\right) d t+m\left|f^{\prime}(b)\right|^{q} \int_{0}^{1} h\left(\frac{2^{\alpha}-t^{\alpha}}{2^{\alpha}}\right) d t\right.\right. \\
& \left.\quad-c m(b-a)^{2} \int_{0}^{1} h\left(\frac{t^{\alpha}}{2^{\alpha}}\right) h\left(\frac{2^{\alpha}-t^{\alpha}}{2^{\alpha}}\right) d t\right)^{\frac{1}{q}} \\
& \quad+\left(m\left|f^{\prime}\left(\frac{a}{m^{2}}\right)\right|^{q} \int_{0}^{1} h\left(\frac{2^{\alpha}-t^{\alpha}}{2^{\alpha}}\right) d t+\left|f^{\prime}(b)\right|^{q} \int_{0}^{1} h\left(\frac{t^{\alpha}}{2^{\alpha}}\right) d t-c m\left(b-\frac{a}{m^{2}}\right)^{2}\right. \\
& \left.\left.\quad \times \int_{0}^{1} h\left(\frac{t^{\alpha}}{2^{\alpha}}\right) h\left(\frac{2^{\alpha}-t^{\alpha}}{2^{\alpha}}\right) d t\right)^{\frac{1}{q}}\right] .
\end{aligned}
$$

\section{Remark 7}

(i) If $\alpha=m=1$ and $h(t)=t$ in (3.7), we get the inequality stated in [10, Theorem 13].

(ii) If $\alpha=1, h(t)=t$ and take $\psi$ as the identity function in (3.7), we get the inequality stated in [5, Theorem 10].

(iii) If $\alpha=1, h(t)=t, c=0$ and take $\psi$ as the identity function in (3.7), we get the inequality stated in [7, Theorem 2.7].

(iv) If $\alpha=\mu=m=1, h(t)=t, c=0$ and take $\psi$ as the identity function in inequality (3.7), we get the inequality stated in [16, Theorem 2.4]. 
Corollary 10 If $\alpha=1$ in (3.7), then the following inequality holds for strongly $(h-m)$ convex function:

$$
\begin{aligned}
& \mid \frac{2^{\mu-1} \Gamma(\mu+1)}{(m b-a)^{\mu}}\left[I_{\psi^{-1}\left(\frac{a+m b}{2}\right)^{+}}^{\mu, \psi}(f \circ \psi)\left(\psi^{-1}(m b)+m^{\mu+1} I_{\psi^{-1}\left(\frac{a+m b}{2 m}\right)^{-}}^{\mu, \psi}(f \circ \psi)\left(\psi^{-1}\left(\frac{a}{m}\right)\right)\right]\right. \\
& \quad-\frac{1}{2}\left[f\left(\frac{a+m b}{2}\right)+m f\left(\frac{a+m b}{2 m}\right)\right] \mid \\
& \leq \frac{m b-a}{4(\mu p+1)^{\frac{1}{p}}}\left[\left(\left|f^{\prime}(a)\right|^{q} \int_{0}^{1} h\left(\frac{t}{2}\right) d t+m\left|f^{\prime}(b)\right|^{q} \int_{0}^{1} h\left(\frac{2-t}{2}\right) d t\right.\right. \\
& \left.\quad-c m(b-a)^{2} \int_{0}^{1} h\left(\frac{t}{2}\right) h\left(\frac{2-t}{2}\right) d t\right)^{\frac{1}{q}} \\
& \quad+\left(m\left|f^{\prime}\left(\frac{a}{m^{2}}\right)\right|_{0}^{q} \int_{0}^{1} h\left(\frac{2-t}{2}\right) d t+\left|f^{\prime}(b)\right|^{q} \int_{0}^{1} h\left(\frac{t}{2}\right) d t\right. \\
& \left.\left.\quad-c m\left(b-\frac{a}{m^{2}}\right)^{2} \int_{0}^{1} h\left(\frac{t}{2}\right) h\left(\frac{2-t}{2}\right)\right)^{\frac{1}{q}}\right] .
\end{aligned}
$$

For $c=0$ in the above inequality, the result for $(h-m)$-convex function can be obtained. Moreover, if $\psi$ is the identity function in the above inequality, then the result for $(\alpha, h-m)$ convex function for Riemann-Liouville fractional integrals can be obtained.

Corollary 11 If $\alpha=1$ and $h(t)=t$ in (3.7), then the following inequality holds for strongly m-convex function:

$$
\begin{aligned}
& \mid \frac{2^{\mu-1} \Gamma(\mu+1)}{(m b-a)^{\mu}}\left[I_{\psi^{-1}\left(\frac{a+m b}{2}\right)^{+}}^{\mu, \psi}(f \circ \psi)\left(\psi^{-1}(m b)+m^{\mu+1} I_{\psi^{-1}\left(\frac{a+m b}{2 m}\right)^{-}}^{\mu, \psi}(f \circ \psi)\left(\psi^{-1}\left(\frac{a}{m}\right)\right)\right]\right. \\
& \quad-\frac{1}{2}\left[f\left(\frac{a+m b}{2}\right)+m f\left(\frac{a+m b}{2 m}\right)\right] \mid \\
& \leq \frac{m b-a}{4^{2-\frac{1}{p}}(\mu p+1)^{\frac{1}{p}}}\left[\left(\left(\left|f^{\prime}(a)\right|+(3 m)^{\frac{1}{q}}\left|f^{\prime}(b)\right|\right)^{q}-\frac{2 c m(b-a)^{2}}{3}\right)^{\frac{1}{q}}\right. \\
& \left.\quad+\left(\left((3 m)^{\frac{1}{q}}\left|f^{\prime}\left(\frac{a}{m^{2}}\right)\right|+\left|f^{\prime}(b)\right|\right)^{q}-\frac{c m\left(b-\frac{a}{m^{2}}\right)^{2}}{3}\right)^{\frac{1}{q}}\right] .
\end{aligned}
$$

If $c=0$ in the above inequality, then the result for m-convex function can be obtained.

\section{$4 k$-Fractional versions of Hadamard inequalities for strongly $(\alpha, h-m)$-convex function and their error estimations}

In this section, we present $k$-fractional versions of Hadamard inequalities and their error estimations discussed in Sect. 2 and Sect. 3.

Theorem 9 Under the assumptions of Theorem 4, the following $k$-fractional integral inequality holds:

$$
f\left(\frac{a+m b}{2}\right)+\frac{c m h\left(\frac{1}{2^{\alpha}}\right) h\left(\frac{2^{\alpha}-1}{2^{\alpha}}\right)}{(\mu+k)(\mu+2 k)}\left[\mu(\mu+k)(b-a)^{2}+2 k^{2}\left(\frac{a}{m}-m b\right)^{2}\right.
$$




$$
\begin{aligned}
& \left.+2 k \mu(b-a)\left(\frac{a}{m}-m b\right)\right] \\
\leq & \frac{\Gamma_{k}(\mu+k)}{(m b-a)^{\frac{\mu}{k}}}\left[h\left(\frac{1}{2^{\alpha}}\right) k^{I_{\psi^{-1}(a)^{+}}^{\mu, \psi}}(f \circ \psi)\left(\psi^{-1}(m b)\right)\right. \\
& \left.+h\left(\frac{2^{\alpha}-1}{2^{\alpha}}\right) m^{\frac{\mu}{k}+1}{ }_{k} I_{\psi^{-1}(b)^{-}}^{\mu, \psi}(f \circ \psi)\left(\psi^{-1}\left(\frac{a}{m}\right)\right)\right] \\
\leq & \frac{\mu}{k}\left[h\left(\frac{1}{2^{\alpha}}\right) f(a)+m h\left(\frac{2^{\alpha}-1}{2^{\alpha}}\right) f(b)\right] \int_{0}^{1} h\left(t^{\alpha}\right) t^{\frac{\mu}{k}-1} d t \\
& +\frac{m \mu}{k}\left(h\left(\frac{1}{2^{\alpha}}\right) f(b)+m h\left(\frac{2^{\alpha}-1}{2^{\alpha}}\right) f\left(\frac{a}{m^{2}}\right)\right) \\
& \times \int_{0}^{1} t^{\frac{\mu}{k}-1} h\left(1-t^{\alpha}\right) d t-\frac{c m \mu}{k}\left[h\left(\frac{1}{2^{\alpha}}\right)(b-a)^{2}+m h\left(\frac{2^{\alpha}-1}{2^{\alpha}}\right)\left(b-\frac{a}{m^{2}}\right)^{2}\right] \\
& \times \int_{0}^{1} t^{\frac{\mu}{k}-1} h\left(t^{\alpha}\right) h\left(1-t^{\alpha}\right) d t
\end{aligned}
$$

with $\mu, k>0$.

Proof Using (1.14) and (1.15) after replacing $\mu$ with $\frac{\mu}{k}$ in (2.1), we get inequality (4.1).

Corollary 12 If $\alpha=1$ in (4.1), then the following $k$-fractional integral inequality holds for strongly $(h-m)$-convex function:

$$
\begin{aligned}
& f\left(\frac{a+m b}{2}\right)+\frac{c m h^{2}\left(\frac{1}{2}\right)}{(\mu+k)(\mu+2 k)}\left[\mu(\mu+k)(b-a)^{2}+2 k^{2}\left(\frac{a}{m}-m b\right)^{2}\right. \\
& \left.+2 k \mu(b-a)\left(\frac{a}{m}-m b\right)\right] \\
& \leq \frac{h\left(\frac{1}{2}\right) \Gamma_{k}(\mu+k)}{(m b-a)^{\frac{\mu}{k}}}\left[{ }_{k} I_{\psi^{-1}(a)^{+}}^{\mu, \psi}(f \circ \psi)\left(\psi^{-1}(m b)\right)+m^{\frac{\mu}{k}+1}{ }_{k} I_{\psi^{-1}(b)^{-}}^{\mu, \psi}(f \circ \psi)\left(\psi^{-1}\left(\frac{a}{m}\right)\right)\right] \\
& \leq \frac{h\left(\frac{1}{2}\right) \mu[f(a)+m f(b)]}{k} \int_{0}^{1} h(t) t^{\frac{\mu}{k}-1} d t \\
& +\frac{m h\left(\frac{1}{2}\right) \mu}{k}\left[f(b)+m f\left(\frac{a}{m^{2}}\right)\right] \int_{0}^{1} t^{\frac{\mu}{k}-1} h(1-t) d t \\
& -\frac{c m h\left(\frac{1}{2}\right) \mu}{k}\left[(b-a)^{2}+m\left(b-\frac{a}{m^{2}}\right)^{2}\right] \int_{0}^{1} h(t) h(1-t) t^{\frac{\mu}{k}-1} d t .
\end{aligned}
$$

Corollary 13 If $\alpha=1$ and $h(t)=t$ in (4.1), then the following inequality holds for strongly m-convex function:

$$
\begin{aligned}
f\left(\frac{a+m b}{2}\right)+\frac{c m}{4(\mu+k)(\mu+2 k)}\left[\mu(\mu+k)(b-a)^{2}+2 k^{2}\left(\frac{a}{m}-m b\right)^{2}\right. \\
\left.\quad+2 k \mu(b-a)\left(\frac{a}{m}-m b\right)\right] \\
\leq \frac{\Gamma_{k}(\mu+k)}{2(m b-a)^{\frac{\mu}{k}}}\left[{ }_{k} I_{\psi^{-1}(a)^{+}}^{\mu, \psi}(f \circ \psi)\left(\psi^{-1}(m b)\right)+m^{\frac{\mu}{k}+1}{ }_{k} I_{\psi^{-1}(b)^{-}}^{\mu,}(f \circ \psi)\left(\psi^{-1}\left(\frac{a}{m}\right)\right)\right]
\end{aligned}
$$




$$
\begin{aligned}
\leq & \frac{\mu[f(a)+m f(b)]}{2(\mu+k)}+\frac{m k}{2(\mu+k)}\left[f(b)+m f\left(\frac{a}{m^{2}}\right)\right] \\
& -\frac{c m \mu k}{2(\mu+k)(\mu+2 k)}\left[(b-a)^{2}+m\left(b-\frac{a}{m^{2}}\right)^{2}\right] .
\end{aligned}
$$

Theorem 10 Under the assumptions of Theorem 4, the following $k$-fractional integral inequality holds:

$$
\begin{aligned}
f\left(\frac{a}{2}\right)+m b & +\frac{c m \mu h\left(\frac{1}{2^{\alpha}}\right) h\left(\frac{2^{\alpha}-1}{2^{\alpha}}\right)}{4(\mu+2 k)}\left[\mu(\mu+k)(b-a)^{2}\right. \\
& +\left(\frac{a}{m}-m b\right)^{2}\left(\mu^{2}+5 k \mu+8 k^{2}\right) \\
& \left.+2 \mu(b-a)\left(\frac{a}{m}-m b\right)(\mu+3 k)\right] \\
\leq & \frac{2^{\frac{\mu}{k}} \Gamma_{k}(\mu+k)}{(m b-a)^{\frac{\mu}{k}}}\left[h\left(\frac{1}{2^{\alpha}}\right){ }_{k} I_{\psi^{-1}\left(\frac{a+m b}{2}\right)^{+}}(f \circ \psi)\left(\psi^{-1}(m b)\right)\right. \\
& \left.\left.+m^{\frac{\mu}{k}+1} h\left(\frac{2^{\alpha}-1}{2^{\alpha}}\right){ }_{k} I_{\psi^{-1}\left(\frac{a+m b}{2 m}\right)^{-}}^{\mu,} \circ \psi\right)\left(\psi^{-1}\left(\frac{a}{m}\right)\right)\right] \\
\leq & \frac{\mu}{k}\left[h\left(\frac{1}{2^{\alpha}}\right) f(a)+m h\left(\frac{2^{\alpha}-1}{2^{\alpha}}\right) f(b)\right] \int_{0}^{1} h\left(\frac{t^{\alpha}}{2^{\alpha}}\right) t^{\frac{\mu}{k}-1} d t \\
& +\frac{\mu m}{k}\left[h\left(\frac{1}{2^{\alpha}}\right) f(b)+m h\left(\frac{2^{\alpha}-1}{2^{\alpha}}\right) f\left(\frac{a}{m^{2}}\right)\right] \int_{0}^{1} h\left(\frac{2^{\alpha}-t^{\alpha}}{2^{\alpha}}\right) t^{\frac{\mu}{k}-1} d t \\
& -\frac{\mu c m}{k}\left[h\left(\frac{2^{\alpha}-1}{2^{\alpha}}\right)(b-a)^{2}+m h\left(\frac{2^{\alpha}-1}{2^{\alpha}}\right)\left(b-\frac{a}{m^{2}}\right)^{2}\right] \\
& \times \int_{0}^{1} t^{\frac{\mu}{k}-1} h\left(\frac{t^{\alpha}}{2^{\alpha}}\right) h\left(\frac{2^{\alpha}-t^{\alpha}}{2^{\alpha}}\right) d t
\end{aligned}
$$

with $\mu, k>0$.

Proof Using (1.14) and (1.15) after replacing $\mu$ with $\frac{\mu}{k}$ in (2.6), we get inequality (4.2).

Corollary 14 If $\alpha=1$ in (4.2), then the following $k$-fractional integral inequality holds for strongly $(h-m)$-convex functions:

$$
\begin{aligned}
& f\left(\frac{a+m b}{2}\right)+\frac{c m h^{2}\left(\frac{1}{2}\right)}{4(\mu+k)(\mu+2 k)}\left[\mu(\mu+k)(b-a)^{2}+\left(\mu^{2}+5 k \mu+8 k^{2}\right)\left(\frac{a}{m}-m b\right)^{2}\right. \\
& \left.\quad+2 \mu(\mu+3 k)(b-a)\left(\frac{a}{m}-m b\right)\right] \\
& \leq \frac{h\left(\frac{1}{2}\right) 2^{\frac{\mu}{k}} \Gamma_{k}(\mu+k)}{(m b-a)^{\frac{\mu}{k}}}\left[k^{\mu} \psi_{\psi^{-1}\left(\frac{a+m b}{2}\right)^{+}}^{\mu}(f \circ \psi)\left(\psi^{-1}(m b)\right)\right. \\
& \left.\quad+m^{\frac{\mu}{k}+1}{ }_{k} I_{\psi^{-1}\left(\frac{a+m b}{2 m}\right)^{-}}^{\mu}(f \circ \psi)\left(\psi^{-1}\left(\frac{a}{m}\right)\right)\right] \\
& \leq \frac{h\left(\frac{1}{2}\right) \mu}{k}[f(a)+m f(b)] \int_{0}^{1} h\left(\frac{t}{2}\right) t^{\frac{\mu}{k}-1} d t
\end{aligned}
$$




$$
\begin{aligned}
& +\frac{h\left(\frac{1}{2}\right) \mu m}{k}\left[f(b)+m f\left(\frac{a}{m^{2}}\right)\right] \int_{0}^{1} h\left(\frac{2-t}{2}\right) t^{\frac{\mu}{k}-1} d t \\
& -c m h\left(\frac{1}{2}\right)\left[(b-a)^{2}+\left(b-\frac{a}{m^{2}}\right)^{2}\right] \int_{0}^{1} h\left(\frac{t}{2}\right) h\left(\frac{2-t}{2}\right) t^{\frac{\mu}{k}-1} d t
\end{aligned}
$$

Corollary 15 If $\alpha=1$ and $h(t)=t$ in (4.2), then the following inequality holds for $m$-convex function:

$$
\begin{aligned}
f( & \left.\frac{a+m b}{2}\right)+\frac{c m}{16(\mu+k)(\mu+2 k)}\left[\mu(\mu+k)(b-a)^{2}+\left(\mu^{2}+5 k \mu+8 k^{2}\right)\left(\frac{a}{m}-m b\right)^{2}\right. \\
& \left.+2 \mu(\mu+3 k)(b-a)\left(\frac{a}{m}-m b\right)\right] \\
\leq & \frac{2^{\frac{\mu}{k}-1} \Gamma_{k}(\mu+k)}{(m b-a)^{\frac{\mu}{k}}}\left[{ }_{k} I_{\psi^{-1}\left(\frac{a+m b}{2}\right)^{+}}(f \circ \psi)\left(\psi^{-1}(m b)\right)\right. \\
& \left.+m^{\frac{\mu}{k}+1}{ }_{k} I_{\psi^{-1}\left(\frac{a+m b}{2 m}\right)^{-}}(f \circ \psi)\left(\psi^{-1}\left(\frac{a}{m}\right)\right)\right] \\
\leq & \frac{\mu[f(a)+m f(b)]}{4(\mu+k)}+\frac{m(\mu+2 k)}{4(\mu+k)}\left[f(b)+m f\left(\frac{a}{m^{2}}\right)\right] \\
& -\frac{c m \mu(\mu+3 k)}{8(\mu+k)(\mu+2 k)}\left[(b-a)^{2}+\left(b-\frac{a}{m^{2}}\right)^{2}\right] .
\end{aligned}
$$

Theorem 11 Under the assumptions of Theorem 6, the following k-fractional integral inequality holds:

$$
\begin{aligned}
& \left|\frac{f(a)+f(b)}{2}-\frac{\Gamma_{k}(\mu+k)}{2(b-a)^{\frac{\mu}{k}}}\left[k_{\psi^{-1}(a)^{+}}^{\mu, \psi}(f \circ \psi)\left(\psi^{-1}(b)\right)+{ }_{k} I_{\psi^{-1}(b)^{-}}^{\mu, \psi}(f \circ \psi)\left(\psi^{-1}(a)\right)\right]\right| \\
& \leq \frac{b-a}{2}\left[\left|f^{\prime}(a)\right|\left(\int_{0}^{\frac{1}{2}} h\left(t^{\alpha}\right)\left((1-t)^{\frac{\mu}{k}}-t^{\frac{\mu}{k}}\right) d t+\int_{\frac{1}{2}}^{1} h\left(t^{\alpha}\right)\left(t^{\frac{\mu}{k}}-(1-t)^{\frac{\mu}{k}}\right) d t\right)\right. \\
& \quad+m\left|f^{\prime}\left(\frac{b}{m}\right)\right|\left(\int_{0}^{\frac{1}{2}} h\left(1-t^{\alpha}\right)\left((1-t)^{\frac{\mu}{k}}-t^{\frac{\mu}{k}}\right) d t+\int_{\frac{1}{2}}^{1} h\left(1-t^{\alpha}\right)\left(t^{\frac{\mu}{k}}-(1-t)^{\frac{\mu}{k}}\right) d t\right) \\
& \quad-c m\left(\frac{b}{m}-a\right)^{2}\left(\int_{0}^{\frac{1}{2}} h\left(t^{\alpha}\right) h\left(1-t^{\alpha}\right)\left((1-t)^{\frac{\mu}{k}}-t^{\frac{\mu}{k}}\right) d t\right. \\
& \left.\left.\quad+\int_{\frac{1}{2}}^{1} h\left(t^{\alpha}\right) h\left(1-t^{\alpha}\right)\left(t^{\frac{\mu}{k}}-(1-t)^{\frac{\mu}{k}}\right) d t\right)\right]
\end{aligned}
$$

with $\mu, k>0$.

Proof Using (1.14) and (1.15) after replacing $\mu$ with $\frac{\mu}{k}$ in (3.2), we get inequality (4.3).

Corollary 16 If $\alpha=1$ in (4.3), then the following $k$-fractional integral inequality holds for strongly $(h-m)$-convex functions:

$$
\begin{aligned}
& \left|\frac{f(a)+f(b)}{2}-\frac{\Gamma_{k}(\mu+k)}{2(b-a)^{\frac{\mu}{k}}}\left[{ }_{k} I_{\psi^{-1}(a)^{+}}^{\mu, \psi}(f \circ \psi)\left(\psi^{-1}(b)\right)+{ }_{k} I_{\psi^{-1}(b)^{-}}^{\mu, \psi}(f \circ \psi)\left(\psi^{-1}(a)\right)\right]\right| \\
& \quad \leq \frac{b-a}{2}\left[\left|f^{\prime}(a)\right|\left(\int_{0}^{\frac{1}{2}} h(t)\left((1-t)^{\frac{\mu}{k}}-t^{\frac{\mu}{k}}\right) d t+\int_{\frac{1}{2}}^{1} h(t)\left(t^{\frac{\mu}{k}}-(1-t)^{\frac{\mu}{k}}\right) d t\right)\right.
\end{aligned}
$$




$$
\begin{aligned}
& +m\left|f^{\prime}\left(\frac{b}{m}\right)\right|\left(\int_{0}^{\frac{1}{2}} h(1-t)\left((1-t)^{\frac{\mu}{k}}-t^{\frac{\mu}{k}}\right) d t+\int_{\frac{1}{2}}^{1} h(1-t)\left(t^{\frac{\mu}{k}}-(1-t)^{\frac{\mu}{k}}\right) d t\right) \\
& -c m(b-a)^{2}\left(\int_{0}^{\frac{1}{2}} h(t) h(1-t)\left((1-t)^{\frac{\mu}{k}}-t^{\frac{\mu}{k}}\right) d t\right. \\
& \left.\left.-\int_{\frac{1}{2}}^{1} h(t) h(1-t)\left(t^{\frac{\mu}{k}}-(1-t)^{\frac{\mu}{k}}\right) d t\right)\right] .
\end{aligned}
$$

Corollary 17 If $\alpha=1$ and $h(t)=t$ in (4.3), then the following inequality holds for strongly m-convex function:

$$
\begin{aligned}
& \left|\frac{f(a)+f(b)}{2}-\frac{\Gamma_{k}(\mu+k)}{2(b-a)^{\frac{\mu}{k}}}\left[{ }_{k} I_{\psi^{-1}(a)^{+}}^{\mu, \psi}(f \circ \psi)\left(\psi^{-1}(b)\right)+{ }_{k} I_{\psi^{-1}(b)^{-}}^{\mu, \psi}(f \circ \psi)\left(\psi^{-1}(a)\right)\right]\right| \\
& \leq \frac{b-a}{2\left(\frac{\mu}{k}+1\right)}\left(1-\frac{1}{2^{\frac{\mu}{k}}}\right)\left(\left|f^{\prime}(a)\right|+m\left|f^{\prime}\left(\frac{b}{m}\right)\right|\right)-\frac{c\left(\frac{b}{m}-a\right)^{3}\left(1-\frac{\frac{\mu}{k}+4}{2^{\frac{\mu}{k}+2}}\right)}{\left(\frac{\mu}{k}+2\right)\left(\frac{\mu}{k}+3\right)}
\end{aligned}
$$

Theorem 12 Under the assumptions of Theorem 7, the following k-fractional integral inequality holds:

$$
\begin{aligned}
& \mid \frac{2^{\frac{\mu}{k}-1} \Gamma_{k}(\mu+k)}{(m b-a)^{\frac{\mu}{k}}}\left[{ }_{k} I_{\psi^{-1}\left(\frac{a+m b}{2}\right)^{+}}^{\mu, \psi}(f \circ \psi)\left(\psi^{-1}(m b)\right)\right. \\
& \left.+m^{\frac{\mu}{k}+1}{ }_{k} I_{\psi^{-1}\left(\frac{a+m b}{2 m}\right)^{-}}^{\mu, \psi}(f \circ \psi)\left(\psi^{-1}\left(\frac{a}{m}\right)\right)\right] \\
& -\frac{1}{2}\left[f\left(\frac{a+m b}{2}\right)+m f\left(\frac{a+m b}{2 m}\right)\right] \mid \\
& \leq \frac{m b-a}{4\left(\frac{\mu}{k}+1\right)^{\frac{1}{q}-1}}\left[\left(\left|f^{\prime}(a)\right|^{q} \int_{0}^{1} h\left(\frac{t^{\alpha}}{2^{\alpha}}\right) t^{\frac{\mu}{k}} d t+m\left|f^{\prime}(b)\right|^{q} \int_{0}^{1} h\left(\frac{2^{\alpha}-t^{\alpha}}{2^{\alpha}}\right) t^{\frac{\mu}{k}} d t\right.\right. \\
& \left.-c m(b-a)^{2} \int_{0}^{1} h\left(\frac{t^{\alpha}}{2^{\alpha}}\right) h\left(\frac{2^{\alpha}-t^{\alpha}}{2^{\alpha}}\right) t^{\frac{\mu}{k}} d t\right)^{\frac{1}{q}} \\
& +\left(m\left|f^{\prime}\left(\frac{a}{m^{2}}\right)\right|^{q} \int_{0}^{1} h\left(\frac{2^{\alpha}-t^{\alpha}}{2^{\alpha}}\right) t^{\frac{\mu}{k}} d t+\left|f^{\prime}(b)\right|^{q} \int_{0}^{1} h\left(\frac{t^{\alpha}}{2^{\alpha}}\right) t^{\frac{\mu}{k}} d t\right. \\
& \left.\left.-c m\left(b-\frac{a}{m^{2}}\right)^{2} \int_{0}^{1} h\left(\frac{t^{\alpha}}{2^{\alpha}}\right) h\left(\frac{2^{\alpha}-t^{\alpha}}{2^{\alpha}}\right) t^{\frac{\mu}{k}} d t\right)^{\frac{1}{q}}\right]
\end{aligned}
$$

with $\mu, k>0$.

Proof Using (1.14) and (1.15) after replacing $\mu$ with $\frac{\mu}{k}$ in (3.6), we get inequality (4.4).

Corollary 18 If $\alpha=1$ in (4.4), then the following $k$-fractional integral inequality holds for strongly $(h-m)$-convex function:

$$
\begin{array}{r}
\mid \frac{2^{\frac{\mu}{k}-1} \Gamma_{k}(\mu+k)}{(m b-a)^{\frac{\mu}{k}}}\left[{ }_{k} I_{\psi^{-1}\left(\frac{a+m b}{2}\right)^{+}}^{\mu, \psi}(f \circ \psi)\left(\psi^{-1}(m b)\right)\right. \\
\left.\quad+m^{\frac{\mu}{k}+1}{ }_{k} I_{\psi^{-1}\left(\frac{a+m b}{2 m}\right)^{-}}^{\mu, \psi}(f \circ \psi)\left(\psi^{-1}\left(\frac{a}{m}\right)\right)\right]
\end{array}
$$




$$
\begin{aligned}
& -\frac{1}{2}\left[f\left(\frac{a+m b}{2}\right)+m f\left(\frac{a+m b}{2 m}\right)\right] \mid \\
\leq & \frac{m b-a}{4\left(\frac{\mu}{k}+1\right)^{\frac{1}{q}-1}}\left[\left(\left|f^{\prime}(a)\right|^{q} \int_{0}^{1} h\left(\frac{t}{2}\right) t^{\frac{\mu}{k}} d t+m\left|f^{\prime}(b)\right|^{q} \int_{0}^{1} h\left(\frac{2-t}{2}\right) t^{\frac{\mu}{k}} d t\right.\right. \\
& \left.-c m(b-a)^{2} \int_{0}^{1} h\left(\frac{t}{2}\right) h\left(\frac{2-t}{2}\right) t^{\frac{\mu}{k}} d t\right)^{\frac{1}{q}} \\
& +\left(m\left|f^{\prime}\left(\frac{a}{m^{2}}\right)\right|^{q} \int_{0}^{1} h\left(\frac{2-t}{2}\right) t^{\frac{\mu}{k}} d t+\left|f^{\prime}(b)\right|^{q} \int_{0}^{1} h\left(\frac{t}{2}\right) t^{\frac{\mu}{k}} d t-c m\left(b-\frac{a}{m^{2}}\right)^{2}\right. \\
& \left.\left.\times \int_{0}^{1} h\left(\frac{t}{2}\right) h\left(\frac{2-t}{2}\right) t^{\frac{\mu}{k}} d t\right)^{\frac{1}{q}}\right] .
\end{aligned}
$$

Corollary 19 If $\alpha=1$ and $h(t)=t$ in (4.4), then the following $k$-fractional integral inequality holds for strongly m-convex function:

$$
\begin{aligned}
& \mid \frac{2^{\frac{\mu}{k}-1} \Gamma_{k}(m u+k)}{(m b-a)^{\frac{\mu}{k}}}\left[{ }_{k} I_{\psi^{-1}\left(\frac{a+m b}{2}\right)^{+}}^{\mu, \psi}(f \circ \psi)\left(\psi^{-1}(m b)\right)\right. \\
& \left.\quad+m^{\frac{\mu}{k}+1}{ }_{k} I_{\psi^{-1}\left(\frac{a+m b}{2 m}\right)^{-}}^{\mu, \psi}(f \circ \psi)\left(\psi^{-1}\left(\frac{a}{m}\right)\right)\right] \\
& \quad-\frac{1}{2}\left[f\left(\frac{a+m b}{2}\right)+m f\left(\frac{a+m b}{2 m}\right)\right] \mid \\
& \leq \quad \frac{m b-a}{4\left(\frac{\mu}{k}+1\right)^{\frac{1}{q}-1}\left[\left(\left|f^{\prime}(a)\right|^{q}\left(\frac{\mu}{k}+1\right)+m\left|f^{\prime}(b)\right|^{q}\left(\frac{\mu}{k}+3\right)-\frac{c m(b-a)^{2}\left(\frac{\mu}{k}+1\right)\left(\frac{\mu}{k}+4\right)}{2\left(\frac{\mu}{k}+3\right)}\right)^{\frac{1}{q}}\right.} \\
& \quad+\left(m\left|f^{\prime}\left(\frac{a}{m^{2}}\right)\right|^{q}\left(\frac{\mu}{k}+3\right)+\left|f^{\prime}(b)\right|^{q}\left(\frac{\mu}{k}+1\right)\right. \\
& \left.\left.\quad-\frac{c m\left(b-\frac{a}{m^{2}}\right)^{2}\left(\frac{\mu}{k}+1\right)\left(\frac{\mu}{k}+4\right)}{2\left(\frac{\mu}{k}+3\right)}\right)^{\frac{1}{q}}\right] .
\end{aligned}
$$

Theorem 13 Under the assumptions of Theorem 8, the following k-fractional integral inequality holds:

$$
\begin{aligned}
& \mid \frac{2^{\frac{\mu}{k}-1} \Gamma_{k}(\mu+k)}{(m b-a)^{\frac{\mu}{k}}}\left[{ }_{k} I_{\psi^{-1}\left(\frac{a+m b}{2}\right)^{+}}^{\mu, \psi}(f \circ \psi)\left(\psi^{-1}(m b)\right)\right. \\
& \left.\quad+m^{\frac{\mu}{k}+1}{ }_{k} I_{\psi^{-1}\left(\frac{a+m b}{2 m}\right)^{-}}^{\mu, \psi}(f \circ \psi)\left(\psi^{-1}\left(\frac{a}{m}\right)\right)\right] \\
& \quad-\frac{1}{2}\left[f\left(\frac{a+m b}{2}\right)+m f\left(\frac{a+m b}{2 m}\right)\right] \mid \\
& \leq \frac{m b-a}{4\left(\frac{\mu p}{k}+1\right)^{\frac{1}{p}}}\left[\left(\left|f^{\prime}(a)\right|^{q} \int_{0}^{1} h\left(\frac{t^{\alpha}}{2^{\alpha}}\right) d t+m\left|f^{\prime}(b)\right|^{q} \int_{0}^{1} h\left(\frac{2^{\alpha}-t^{\alpha}}{2^{\alpha}}\right) d t\right.\right. \\
& \left.\quad-c m(b-a)^{2} \int_{0}^{1} h\left(\frac{t^{\alpha}}{2^{\alpha}}\right) h\left(\frac{2^{\alpha}-t^{\alpha}}{2^{\alpha}}\right) d t\right)^{\frac{1}{q}}
\end{aligned}
$$




$$
\begin{aligned}
& +\left(m\left|f^{\prime}\left(\frac{a}{m^{2}}\right)\right|^{q} \int_{0}^{1} h\left(\frac{2^{\alpha}-t^{\alpha}}{2^{\alpha}}\right) d t+\left|f^{\prime}(b)\right|^{q} \int_{0}^{1} h\left(\frac{t^{\alpha}}{2^{\alpha}}\right) d t\right. \\
& \left.\left.-c m\left(b-\frac{a}{m^{2}}\right)^{2} \int_{0}^{1} h\left(\frac{t^{\alpha}}{2^{\alpha}}\right) h\left(\frac{2^{\alpha}-t^{\alpha}}{2^{\alpha}}\right)\right)^{\frac{1}{q}}\right]
\end{aligned}
$$

with $\mu, k>0$ and $\frac{1}{p}+\frac{1}{q}=1$.

Proof Using (1.14) and (1.15) after replacing $\mu$ with $\frac{\mu}{k}$ in (3.7), we get inequality (4.5).

Corollary 20 If $\alpha=1$ in (4.5), then the following $k$-fractional integral inequality holds for strongly $(h-m)$-convex function:

$$
\begin{aligned}
& \mid \frac{2^{\frac{\mu}{k}-1} \Gamma_{k}(\mu+k)}{(m b-a)^{\frac{\mu}{k}}}\left[{ }_{k} I_{\psi^{-1}\left(\frac{a+m b}{2}\right)^{+}}^{\mu, \psi}(f \circ \psi)\left(\psi^{-1}(m b)\right)\right. \\
& \left.\quad+m^{\frac{\mu}{k}+1}{ }_{k} I_{\psi^{-1}\left(\frac{a+m b}{2 m}\right)^{-}}^{\mu, \psi}(f \circ \psi)\left(\psi^{-1}\left(\frac{a}{m}\right)\right)\right] \\
& \quad-\frac{1}{2}\left[f\left(\frac{a+m b}{2}\right)+m f\left(\frac{a+m b}{2 m}\right)\right] \mid \\
& \leq \frac{m b-a}{4\left(\frac{\mu p}{k}+1\right)^{\frac{1}{p}}}\left[\left(\left|f^{\prime}(a)\right|^{q} \int_{0}^{1} h\left(\frac{t}{2}\right) d t+m\left|f^{\prime}(b)\right|^{q} \int_{0}^{1} h\left(\frac{2-t}{2}\right) d t\right.\right. \\
& \left.\quad-c m(b-a)^{2} \int_{0}^{1} h\left(\frac{t}{2}\right) h\left(\frac{2-t}{2}\right) d t\right) \\
& \quad+\left(m\left|f^{\prime}\left(\frac{a}{m^{2}}\right)\right|^{\frac{1}{q}} h\left(\frac{2-t}{2}\right) d t+\left|f^{\prime}(b)\right|^{q} \int_{0}^{1} h\left(\frac{t}{2}\right) d t\right. \\
& \left.\left.\quad-c m\left(b-\frac{a}{m^{2}}\right)^{2} \int_{0}^{1} h\left(\frac{t}{2}\right) h\left(\frac{2-t}{2}\right) d t\right)^{\frac{1}{q}}\right]
\end{aligned}
$$

Corollary 21 If $\alpha=1$ and $h(t)=t$ in (4.5), then the following $k$-fractional integral inequality holds for strongly m-convex function:

$$
\begin{aligned}
& \mid \frac{2^{\frac{\mu}{k}-1} \Gamma_{k}(\mu+k)}{(m b-a)^{\frac{\mu}{k}}}\left[{ }_{k} I_{\psi^{-1}\left(\frac{a+m b}{2}\right)^{+}}^{\mu, \psi}(f \circ \psi)\left(\psi^{-1}(m b)\right)\right. \\
& \left.\quad+m^{\frac{\mu}{k}+1}{ }_{k} I_{\psi^{-1}\left(\frac{a+m b}{2 m}\right)^{-}}^{\mu, \psi}(f \circ \psi)\left(\psi^{-1}\left(\frac{a}{m}\right)\right)\right] \\
& \quad-\frac{1}{2}\left[f\left(\frac{a+m b}{2}\right)+m f\left(\frac{a+m b}{2 m}\right)\right] \mid \\
& \leq \frac{m b-a}{4^{2-\frac{1}{p}}\left(\frac{\mu p}{k}+1\right)^{\frac{1}{p}}}\left[\left(\left(\left|f^{\prime}(a)\right|^{q}+(3 m)^{\frac{1}{q}}\left|f^{\prime}(b)\right|\right)^{q}-\frac{2 c m(b-a)^{2}}{3}\right)^{\frac{1}{q}}\right. \\
& \left.\quad+\left(\left((3 m)^{\frac{1}{q}}\left|f^{\prime}\left(\frac{a}{m^{2}}\right)\right|+\left|f^{\prime}(b)\right|\right)^{q}-\frac{2 c m\left(b-\frac{a}{m^{2}}\right)^{2}}{3}\right)^{\frac{1}{q}}\right] .
\end{aligned}
$$

\section{Conclusion}

This article deals with Hadamard inequalities for strongly $(\alpha, h-m)$-convex function via generalized Riemann-Liouville fractional integrals. The outcomes of this paper pro- 
vide refinements of Hadamard inequalities for different kinds of convex functions. Error bounds of Hadamard inequalities are obtained for differentiable convex functions of several kinds.

\section{Acknowledgements}

This work was supported by the Dong-A University research fund.

\section{Funding}

There is no funding available for the publication of this paper.

Availability of data and materials

There is no additional data required for the finding of results of this paper.

\section{Competing interests}

The authors declare that they have no competing interests.

\section{Authors' contributions}

All authors have equal contribution in this article. All authors read and approved the final manuscript.

\section{Author details}

${ }^{1}$ Department of Mathematics, COMSATS University Islamabad, Attock Campus, Pakistan. ${ }^{2}$ Department of Mathematics, Dong-A University, Busan 49315, Korea. ${ }^{3}$ Department of Mathematics, Faculty of Science and Arts, Kahramanmaras Sutcu Imam University, 46100, Kahramanmaras, Turkey. ${ }^{4}$ Department of Mathematics, Gyeongsang National University, Jinju 52828, Korea. ${ }^{5}$ Center for General Education, China Medical University, Taichung 40402, Taiwan.

\section{Publisher's Note}

Springer Nature remains neutral with regard to jurisdictional claims in published maps and institutional affiliations.

Received: 1 June 2021 Accepted: 10 August 2021 Published online: 23 August 2021

\section{References}

1. Agarwal, P., Tariboon, J., Ntouyas, S.K.: Some generalized Riemann-Liouville k-fractional integral inequalities. J. Inequal. Appl. 2016, 122 (2016)

2. Akkurt, A., Yildirim, M.E., Yildirim, H.: On some integral inequalities for ( $k$, $h$ )-Riemann-Liouville fractional integral. New Trends Math. Sci. 4(2), 138-146 (2016)

3. Dragomir, S.S., Agarwal, R.P.: Two inequalities for differentiable mappings and applications to special means of real numbers and to trapezoidal formula. Appl. Math. Lett. 11(5), 91-95 (1998)

4. Farid, G.: Existence of an integral operator and its consequences in fractional and conformable integrals. Open J. Math. Sci. 3(3), 210-216 (2019)

5. Farid, G., Akbar, S.B., Mishra, L.N., Mishra, V.N.: Riemann-Liouville fractional versions of Hadamard inequality for strongly m-convex functions (submitted)

6. Farid, G., Rehman, A.U., Ain, Q.U.: k-Fractional integral inequalities of Hadamard type for $(h-m)$-convex functions. Comput. Methods Differ. Equ. 8(1), 119-140 (2020)

7. Farid, G., Rehman, A.U., Tariq, B.: On Hadamard-type inequalities for $m$-convex functions via Riemann-Liouville fractional integrals. Stud. Univ. Babeş-Bolyai, Math. 62(2), 141-150 (2017)

8. Farid, G., Rehman, A.U., Tariq, B., Waheed, A.: On Hadamard type inequalities for $m$-convex functions via fractional integrals. J. Inequal. Spec. Funct. 7(4), 150-167 (2016)

9. Farid, G., Yasmeen, H., Ahmad, H., Jung, C.Y.: Riemann-Liouville fractional integral operators with respect to increasing functions and strongly $(\alpha, m)$-convex functions. AIMS Math. 6(10), 11403-11424 (2021)

10. Farid, G., Yasmeen, H., Jung, C.Y., Shim, S.H., Ha, G.: Refinements and generalizations of some fractional integral inequalities via strongly convex functions. Math. Probl. Eng. 2021, Article ID 6667226 (2021)

11. Kadakal, M., İşcan, I.., Agarwal, P., Jeli, M.: Exponential trigonometric convex functions and Hermite-Hadamard type inequalities. Math. Slovaca 71(1), 43-56 (2021)

12. Katugampola, U.N.: New approach to a generalized fractional integral. Appl. Math. Comput. 218(3), 860-865 (2011)

13. Khan, M.A., Begum, S., Khurshid, Y., Chu, Y.M.: Ostrowski type inequalities involving conformable fractional integrals. J. Inequal. Appl. 2018, 70 (2018)

14. Khan, M.A., Wu, S.H., Ullah, H., Chu, Y.M.: Discrete majorization type inequalities for convex functions on rectangles. J. Inequal. Appl. 2019, 16 (2019)

15. Kilbas, A.A., Srivastava, H.M., Trujillo, J.J.: Theory and Applications of Fractional Differential Equations. North-Holland Math. Stud. Elsevier, New York (2006)

16. Kirmaci, U.S.: Inequalities for differentiable mappings and applications to special means of real numbers to midpoint formula. Appl. Math. Comput. 147(1), 137-146 (2004)

17. Kórus, P.: An extension of the Hermite-Hadamard inequality for convex and s-convex functions. Aequ. Math. 93(3), 527-534 (2019)

18. Liu, K., Wang, J., O'Regan, D.: On the Hermite-Hadamard type inequality for $\psi$-Riemann-Liouville fractional integrals via convex functions. J. Inequal. Appl. 2019, 27 (2019)

19. Merentes, N., Nikodem, K.: Remarks on strongly convex functions. Aequ. Math. 80, 193-199 (2010)

20. Miao, C., Farid, G., Yasmeen, H., Bian, Y.: Generalized Hadamard fractional integral inequalities for strongly $(s, m)$-convex functions. J. Math. 2021, Article ID 6642289 (2021) 
21. Miller, K., Ross, B.: An Introduction to the Fractional Calculus and Fractional Differential Equations. Wiley, New York (1993)

22. Mohammed, P.O.: Hermite-Hadamard inequalities for Riemann-Liouville fractional integrals of a convex function with respect to a monotone function. Math. Methods Appl. Sci. 44(3), 2314-2324 (2021)

23. Mubeen, S., Habibullah, G.M.: k-Fractional integrals and applications. Int. J. Contemp. Math. Sci. 7, 89-94 (2012)

24. Ntouyas, S.K., Agarwal, P., Tariboon, J.: On Pólya-Szegö and Chebyshev types inequalities involving the Riemann-Liouville fractional integral operators. J. Math. Inequal. 10(2), 491-504 (2016)

25. Raina, R.K.: On generalized Wright's hypergeometric functions and fractional calculus operators. East Asian Math. J. 21(2), 191-203 (2005)

26. Samko, S.G., Kilbas, A.A., Marichev, O.I.: Fractional Integrals and Derivatives-Theory and Applications. Gordon \& Breach, Linghorne (1993)

27. Sarikaya, M.Z., Set, E., Yaldiz, H., Başak, N.: Hermite-Hadamard's inequalities for fractional integrals and related fractional inequalities. Math. Comput. Model. 57(9-10), 2403-2407 (2013)

28. Sarikaya, M.Z., Yildirim, H.: On Hermite-Hadamard type inequalities for Riemann-Liouville fractional integrals. Miskolc Math. Notes 17(2), 1049-1059 (2017)

29. Set, E., Sardari, M., Ozdemir, M.E., Rooin, J.: On generalizations of the Hadamard inequality for $(\alpha, m)$-convex functions. Kyungpook Math. J. 52(3), 307-317 (2012)

30. Sitho, S., Ntouyas, S.K., Agarwal, P., Tariboon, J.: Noninstantaneous impulsive inequalities via conformable fractional calculus. J. Inequal. Appl. 2018, 261 (2018)

31. Ullah, S.Z., Khan, M.A., Chu, Y.-M.: A note on generalized convex functions. J. Inequal. Appl. 2019, 291 (2019)

32. Wang, M., Zhang, W., Chu, Y.: Monotonicity, convexity and inequalities involving the generalized elliptic integrals. Acta Math. Sci. 39(5), 1440-1450 (2019)

33. Wu, S.: On the weighted generalization of the Hermite-Hadamard inequality and its applications. Rocky Mt. J. Math. 39(5), 1741-1749 (2009)

34. You, X.X., Ali, M.A., Budak, H., Agarwal, P., Chu, Y.M.: Extensions of Hermite-Hadamard inequalities for harmonically convex functions via generalized fractional integrals. J. Inequal. Appl. 2021, 102 (2021)

35. Zhang, Z., Farid, G., Mahreen, K.: Inequalities for unified integral operators via strongly $(\alpha, h-m)$-convexity. J. Funct. Spaces 2021, Article ID 6675826 (2021)

\section{Submit your manuscript to a SpringerOpen ${ }^{\circ}$ journal and benefit from:}

- Convenient online submission

- Rigorous peer review

- Open access: articles freely available online

- High visibility within the field

- Retaining the copyright to your article

Submit your next manuscript at $\boldsymbol{\nabla}$ springeropen.com 